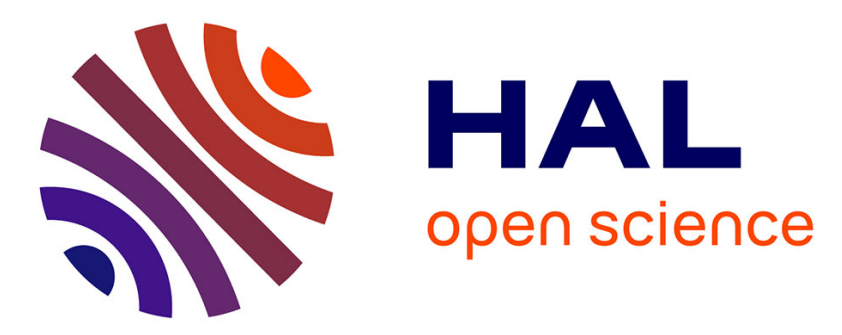

\title{
Conformational Ensemble and Biological Role of the TCTP Intrinsically Disordered Region: Influence of Calcium and Phosphorylation
}

Florian Malard, Nadine Assrir, Mouad Alami, Samir Messaoudi, Ewen Lescop, Tâp Ha-Duong

\section{To cite this version:}

Florian Malard, Nadine Assrir, Mouad Alami, Samir Messaoudi, Ewen Lescop, et al.. Conformational Ensemble and Biological Role of the TCTP Intrinsically Disordered Region: Influence of Calcium and Phosphorylation. Journal of Molecular Biology, 2018, 430 (11), pp.1621-1639. 10.1016/j.jmb.2018.04.024 . hal-02114798

\section{HAL Id: hal-02114798 \\ https://hal.science/hal-02114798}

Submitted on 27 Jul 2021

HAL is a multi-disciplinary open access archive for the deposit and dissemination of scientific research documents, whether they are published or not. The documents may come from teaching and research institutions in France or abroad, or from public or private research centers.
L'archive ouverte pluridisciplinaire HAL, est destinée au dépôt et à la diffusion de documents scientifiques de niveau recherche, publiés ou non, émanant des établissements d'enseignement et de recherche français ou étrangers, des laboratoires publics ou privés. 
« This document is the Accepted Manuscript version of a Published Work that appeared in final form in Journal of Molecular Biology after peer review and technical editing by the publisher. To access the final edited and published work see

https://doi.org/10.1016/j.jmb.2018.04.024» 


\title{
Conformational ensemble and biological role of the TCTP intrinsically disordered region: Influence of calcium and phosphorylation
}

\author{
Florian Malard ${ }^{\mathrm{a}}$, Nadine Assrir ${ }^{\mathrm{a}}$, Mouad Alami ${ }^{\mathrm{b}}$, Samir Messaoudi ${ }^{\mathrm{b}}$, Ewen \\ Lescop $^{\mathrm{a}, *}$, Tâp Ha-Duong ${ }^{\mathrm{b}, *}$ \\ ${ }^{a}$ Institut de Chimie des Substances Naturelles, CNRS UPR 2301, Université \\ Paris-Saclay, 1 avenue de la Terrasse, 91198 Gif-sur-Yvette, France \\ ${ }^{b}$ BIOCIS, Université Paris-Sud, CNRS UMR 8076, Université Paris-Saclay, 5 rue \\ Jean-Baptiste Clément, 92290 Châtenay-Malabry, France
}

\begin{abstract}
The translationally controlled tumor protein (TCTP) is a multifunctional protein that may interact with many other biomolecules, including itself. The experimental determinations of TCTP structure revealed a folded core domain and an intrinsically disordered region, which includes the first highly conserved TCTP signature, but whose role in the protein functions remains to be elucidated. In this work, we combined NMR experiments and MD simulations to characterize the conformational ensemble of the TCTP intrinsically disordered loop, in the presence or not of calcium ions and with or without the phosphorylation of Ser46 and Ser64. Our results show that these changes in the TCTP electrostatic conditions induce significant shifts of its conformational ensemble towards structures more or less extended in which the disordered loop is pulled away or folded against the core domain. Particularly, these conditions impact the transient contacts between the two
\end{abstract}

\footnotetext{
${ }^{*}$ Corresponding authors: ewen.lescop@cnrs.fr and tap.ha-duong@u-psud.fr
} 
highly conserved signatures of the protein. Moreover, both experimental and theoretical data show that the interface of the non-covalent TCTP dimerization involves its second signature which suggests that this region might be involved in protein-protein interaction. We also show that calcium hampers the formation of TCTP dimers, likely by favouring the competitive binding of the disordered loop to the dimerization interface. All together, we propose that the TCTP intrinsically disordered region is involved in remodelling the core domain surface to modulate its accessibility to its partners in response to a variety of cellular conditions.

Keywords:

Nuclear Magnetic Resonance, Molecular Dynamics, Intrinsically Disordered Protein, TCTP-calcium interaction, TCTP dimerization

\section{Graphical abstract}

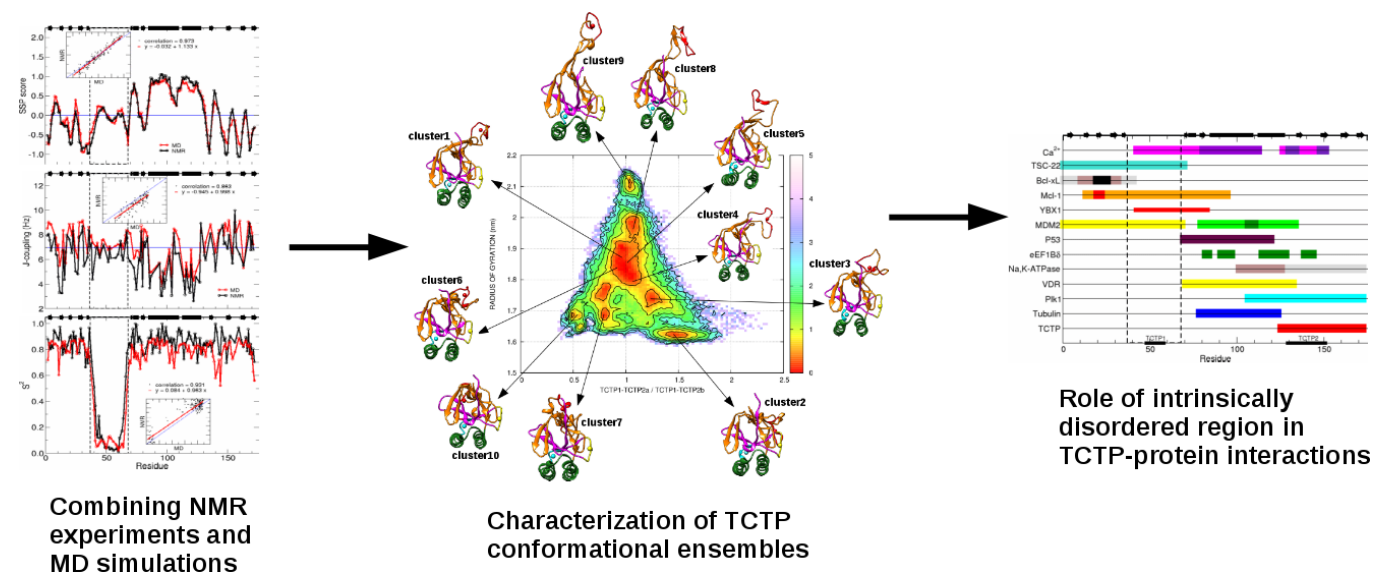




\section{Highlights}

- The role of the TCTP intrinsically disordered region was poorly studied although it contains a highly conserved amino-acid segment.

- NMR experiments and MD simulations show that the disordered loop transiently binds to the TCTP second signature region.

- The presence of calcium or phosphorylation of the disordered loop impact the transient contacts between the two conserved signatures.

- TCTP was shown to form concentration-dependent non-covalent dimers. The interface of dimerization involves its second signature.

- The intrinsically disordered region might modulate the TCTP proteinprotein interactions in response to various cellular conditions.

\section{Introduction}

Abundantly found in eukaryotes, the translationally controlled tumor protein (TCTP) has a very conserved sequence through evolution but a poor amino-acid homology to other proteins. TCTP is actually a multifunctional protein involved in several biological processes, including cell growth, cell division, cell survival, and immune response [1]. It has notably a crucial role in tumorigenesis, and is up-regulated in many cancer cell lines [2]. TCTP is also an important player in the complex process of somatic cell phenotypic reprogramming into embryonic-stem-like cells. Interestingly, it is involved in the tumor reversion process that makes cancer cells lose their malignant 
phenotype $[3,4]$. Thus, TCTP represents a promising biomolecular target for cancer therapy [5].

To exert its various functions, TCTP may interact with many other biomolecules, including itself $[6,7]$. A recent study using coimmunoprecipitation and mass spectrometry identified 98 potential interacting partners for TCTP [8]. At least 33 proteins were previously shown to bind TCTP by yeast two-hybrid screenings [4]. In addition, TCTP is known to sequestrate calcium ions [9-11], presumably to block the $\mathrm{Ca}^{2+}$-dependent apoptosis process [12]. However, very little information on the structures of TCTP-ligand complexes is available, which impedes the full understanding of the mechanisms by which TCTP performs its functions.

Several groups attempted to determine the TCTP binding regions on which its partners interact, using, in most cases, the domain mapping technique that consists in testing if different truncated domains of the protein can bind an identified ligand. To our knowledge, the TCTP binding region of 12 protein partners and calcium ions were reported in the literature (Fig. S1 of the Supporting Information). Despite the limitations of the domain mapping approach, we can roughly distinguish three binding regions, each of them being able to interact with several TCTP partners: the N-terminal region (1-80) encompassing the disordered long loop and the TCTP first signature (TCTP1), the central segment (81-130) which is structured in helical hairpin, and the C-terminal region (131-172) containing the protein second signature TCTP2 (the amino-acid numbering used in this report comes from the human sequence). However, the NMR three-dimensional structures of TCTP $[11,13,14]$ reveal that its N-terminal and C-terminal segments do not 
form two separated domains but are interlaced in a $\beta$-barrel-like architecture whose accessible surface is composed of patches from both N-terminal and C-terminal residues. Besides the fact that truncated segments of TCTP do not necessarily fold like the full-length protein, this makes the domain mapping results even more difficult to be interpreted in terms of protein-protein interfaces. Therefore, experimental and theoretical studies carried out on the native full-length protein should provide more reliable information regarding the TCTP interactome.

Importantly, the highly conserved signature TCTP1 (consensus sequence $\mathrm{IG}[\mathrm{A}-\mathrm{G}] \mathrm{N}[\mathrm{A}-\mathrm{P}] \mathrm{SAE})$ is located in the middle of the protein disordered region (residues 37-68) and probably plays a pivotal role in the TCTP recognition by its multiple partners. To illustrate this, TCTP1 contains the residue Ser53 which was found to be phosphorylated in human cell line during mitosis [15], and can therefore be involved in TCTP functional regulation. Furthermore, it is often highlighted that dynamic intrinsically disordered regions can transiently adopt various secondary structures which are recognized by different ligands [16-18]. Another possible role of the disordered loop is to transiently interact with different parts of the structured core domain, potentially hampering the binding of ligands on these regions (competitive binding) [19-21]. More generally, this loop-core intramolecular interactions could modify the physical-chemical properties of the protein binding surfaces, regulating the binding of other partners. Among the possible binding sites on the TCTP core domain, the second signature TCTP2 is located at the region 129-151 and contains the largely conserved residues Phe134, Phe135, Gly137, Glu138, Met140, Asp143, Tyr151. These solvent-exposed residues delineate an ex- 
tended area on TCTP surface that can be decomposed into two patches formed by residues $129-133$ in strand $\beta 8$ with residues $150-151$ in strand $\beta 9$ (TCTP2a) and by the $\beta 8-\beta 9$ loop residues 138-143 (TCTP2b).

To add complexity to the TCTP structure-activity relationship, residues Ser46 and Ser64, both located in the intrinsically disordered loop, were shown to be hierarchically phosphorylated by the polo-like kinase Plk1 [22]. These post-translational modifications might impact the TCTP conformational ensemble and its binding activity with other proteins. Likewise, the disordered region having many negatively charged residues (Glu40, Asp44, Asp45, Glu55, Glu58, Glu60, Glu63), the binding of $\mathrm{Ca}^{2+}$ ions on TCTP surface is likely to influence the conformational dynamics of its long loop. All these considerations call attention to the possible role of the disordered region in modulating the recognition of TCTP by multiple other proteins. In that context, the present study aims at exploring and characterizing the TCTP conformational ensemble, with a particular focus on its disordered loop. Notably, the impacts of calcium binding and of Ser46 and Ser64 phosphorylation on its structures and surface accessibility will be scrutinized and related to the TCTP activity. For this purpose, we investigated the TCTP structural ensemble under four conditions: no calcium and no phosphorylation (noCanoPhos), with calcium and no phosphorylation (wiCa-noPhos), no calcium and with phosphorylated Ser46 (noCa-pS46), and lastly no calcium and with both Ser46 and Ser64 phosphorylated (noCa-diPhos). 


\section{Results and Discussion}

NMR backbone resonance assignment

The 173-amino-acid construct of the human TCTP used in this study corresponds to the native sequence of the protein with an extra N-terminal Gly residue (Gly0) due to the TEV cleavage site. The ${ }^{1} \mathrm{H}_{-}{ }^{15} \mathrm{~N}$ SOFAST-HMQC spectrum of the protein was of excellent quality with very well dispersed signals in agreement with a properly folded protein. This spectrum was very similar to that previously published [11] with a slightly different TCTP construct (native N-terminal and extra C-terminal Leu and Glu residues) in different experimental conditions. We confirmed and adjusted the backbone resonance assignment using BEST-TROSY type triple resonance correlation experiments [23]. All 173 amino-acids of TCTP except Gly0, Met1, and Asn51 could be assigned to a ${ }^{1} \mathrm{H}^{15}{ }^{15}$ cross-peak. All HSQC cross-peaks could be assigned suggesting that Met1 and Asn51 likely suffer from severe line broadening. The analysis of the secondary structures by TALOS+ software revealed, as expected, a very good agreement between predicted secondary structures and the NMR [11] and crystal [24] structures of the protein.

\section{Analysis of ${ }^{15} \mathrm{~N}$-relaxation}

${ }^{15} \mathrm{~N}$ spin relaxation rates depend mostly on the reorientation of the $\mathrm{N}-\mathrm{H}$ bonds, thus providing rich information about local and global dynamics of the protein. We measured residue-specific ${ }^{15} \mathrm{~N} \mathrm{R}_{1}$ and $\mathrm{R}_{2}$ relaxation rates and $\left\{{ }^{1} \mathrm{H}\right\}-{ }^{15} \mathrm{~N}$ heteronuclear NOE values at $950 \mathrm{MHz}{ }^{1} \mathrm{H}$ frequency, $298 \mathrm{~K}$, and $50 \mu \mathrm{M}$ protein concentration. Extracted parameters show that relaxation rates were very homogeneous along the structured regions of the protein. 
Over the structured parts of TCTP, averaged ${ }^{15} \mathrm{~N} R_{1}, R_{2}$, and $R_{2} / R_{1}$ values were $0.63 \pm 0.07 \mathrm{~s}^{-1}, 18.4 \pm 2.4 \mathrm{~s}^{-1}$ and $29.1 \pm 6.7$, respectively. Within the segment $38-66$, elevated ${ }^{15} \mathrm{~N} \mathrm{R}_{1}$ and decreased values of ${ }^{15} \mathrm{~N} \mathrm{R}_{2}$ and heteronuclear NOE were observed, which is typical of significant mobility in the fast timescale (picosecond to nanosecond). To further analyze ${ }^{15} \mathrm{~N}$-relaxation and $\left\{{ }^{1} \mathrm{H}\right\}-{ }^{15} \mathrm{~N}$ heteronuclear NOE, we computed the global correlation time $\left(\tau_{c}\right)$ of the protein and the residue-specific order parameters $\mathrm{S}^{2}$ by using the Lipari-Szabo formalism [25] and an isotropic rotational diffusion tensor. The apparent correlation time was $10.1 \pm 0.12 \mathrm{~ns}$ which is largely consistent with the value predicted by HYDRONMR [26] for a monomeric TCTP (9.82 ns). The averaged order parameter $\mathrm{S}^{2}$ were $0.86 \pm 0.08$ along the structured parts, in agreement with restricted motions in the core domain of TCTP, whereas much lower values of $\mathrm{S}^{2}$ were obtained within the disordered loop of TCTP, in agreement with its mobility at the ps-ns timescale.

Conformational ensemble of the non-phosphorylated TCTP in the absence of calcium

In this section, the noCa-noPhos TCTP conformational ensemble was first assessed by comparing several observable parameters computed from MD simulations with those measured by NMR experiments (Fig. 1). These include the coupling constants ${ }^{3} \mathrm{~J}_{H_{N}-H_{\alpha}}$ and the N-H order parameter $\mathrm{S}^{2}$ which give information about the protein backbone conformations and dynamics, and secondary chemical shifts which indicate the backbone propensity to form secondary structures. More specifically, we compared the secondary structure propensity (SSP) score that combines the three $\mathrm{C} \alpha, \mathrm{C} \beta$ and $\mathrm{H} \alpha$ secondary chemical shifts as proposed by Marsh et al. [27]. Fig. 1 shows 
that the residue SSP score calculated from MD simulations are in very good agreement with those derived from NMR chemical shifts. Despite a slight offset of the theoretical J-couplings towards higher values when compared to NMR data, the ${ }^{3} \mathrm{~J}_{H_{N}-H_{\alpha}}$ values from simulations correlate well with experimental one. Both SSP score and J-coupling profiles confirm that the region 37-68 has no stable secondary structure, as indicated by their values around 0.0 and $7 \mathrm{~Hz}$, respectively. Finally, the theoretical order parameters $\mathrm{S}^{2}$ appear quite close to those measured by NMR. Notably, in both MD and NMR ensembles, the long loop 37-68 is very mobile, with $\mathrm{S}^{2}$ values below 0.2 for residues 45-65 indicating very large amplitude fluctuations. Interestingly, some residues exhibit lower order parameter in MD than observed by NMR, notably residues Asp11, His77, Glu138, and Asn139 which are in close spatial proximity in the three-dimensional structure, suggesting possible correlated motions on timescales slower than the protein correlation time ( $\sim 10 \mathrm{~ns})$, and hence not detectable by NMR. All together, these comparisons indicate that the TCTP conformational ensemble explored by MD simulations is fairly consistent with the experimental one.

The noCa-noPhos TCTP conformational ensemble was further analyzed to investigate the possible roles of its intrinsically disordered region. We noticed that the long loop containing the signature TCTP1 tends to contact the core domain on the surface defined with the signature TCTP2. In order to gain insight into this propensity, a free energy surface of the protein was computed as a function of its radius of gyration and the ratio of the distance between the centers of mass of segment 48-58 (TCTP1) and of residues 129-133,150-151 (TCTP2a) over the distance between the centers of mass of 
TCTP1 and of residues 138-143 (TCTP2b). The latter parameter was chosen to highlight the inclination of the TCTP first signature to preferentially bind the solvent accessible regions defined by either the patches TCTP2a or TCTP2b. The free energy surface displayed in Fig. 2 shows that the TCTP intrinsically disordered loop can adopt various extended conformations more or less distant from the folded core domain, conferring a protein radius of gyration larger than $1.8 \mathrm{~nm}$. But in addition, this long loop can fold towards the bottom of the core domain resulting in a more compact protein with a radius of gyration lower than $1.8 \mathrm{~nm}$. In the latter case, it is observed that the residues of the TCTP first signature are close to either the residues 129-133,150-151 (TCTP2a) or residues 138-143 (TCTP2b).

To specify these contacts, the fraction of the simulation time for which each residue of the TCTP core domain is distant by less than $3 \AA$ to the disordered region are shown in Fig. 3. It can be seen that the disordered loop is able to transiently but significantly contact the three segments $73-$ 82, 130-142, and 150-158 of the folded core domain. Among these latter, it should be noted that residues His77, Leu78, Gln79, Glu80, Thr81, and Ser82 form with residues Glu138, Asn139, Met140, and Asn141 (TCTP2b) a continuous patch, rather negatively charged, on one side of the protein core domain (Fig. 3). Similarly, residues Lys130, Asn131, Tyr132, and Gln133 form with Asp150 and Tyr151 a continuous area (TCTP2a), at the bottom of the core domain, but less negatively charged due to the presence of the conserved residue Lys130 (Fig. 3). These two patches at the surface of the TCTP core domain are among the most affected areas by the transient binding of its disordered loop. To highlight the residues of the TCTP core domain 
which make contacts with the disordered loop, we first performed a clustering of the TCTP conformations based on their pairwise RMSD, and then, for the ten most populated clusters (Fig. 2), we computed the distance matrix between the residues 48-58 and 129-151. Among the top ten clusters, only cluster2 and cluster10 clearly exhibit contacts between the signature segment TCTP1 and the second signature TCTP2 (Fig. S2 of Supporting Information). In cluster2, the predominant contacts are observed between residues Gly50 and Ala54 of the disordered loop and the residues Phe135, Ile136, and Met140 of the segment TCTP2b. The nature of these interactions is clearly hydrophobic. In cluster10, residues Ile48, Gly49, Gly50, and Asn51 make contacts with the residues Lys130, Asn131, Gln133, and Tyr151 of the region TCTP2a. A visual inspection of cluster10 indicates that, in contrast to cluster2, the core-loop contacts mostly involved polar interactions between the amide group of the Asn51, Asn131, and Gln133 side chains and those of the Gly49, Gly50, and Tyr151 backbone. In none of the TCTP clusters, the signature TCTP1 binds both regions TCTP2a and TCTP2b at the same time.

Calcium binding and impact on TCTP conformational ensemble

As mentioned in the introduction, TCTP is known to sequestrate calcium, but the protein regions that bind the $\mathrm{Ca}^{2+}$ ions are not unambiguously determined. Indeed, using domain mapping techniques, Kim et al. located the $\mathrm{Ca}^{2+}$ binding sites in region 81-112 [10]. With the same approaches, Graidist et al. identified two high affinity and several low affinity binding sites in the regions 43-62, 57-76, and 127-146, with a particular emphasis on residues Glu58 and Glu60 of the TCTP disordered loop [12]. On the other hand, 
Feng et al. found a single weak affinity calcium binding site in the vicinity of residues Asn131, Gln133, and Asp150 by analyzing NMR chemical shift perturbations in TCTP ${ }^{1} \mathrm{H}_{-}{ }^{15} \mathrm{~N}$ SOFAST-HMQC spectra [11]. To resolve these ambiguities, we measured and analyzed sets of calcium-induced chemical shift perturbations in light of MD simulations of the non-phosphorylated TCTP in the presence of calcium (wiCa-noPhos). Before, it should be reminded that ${ }^{1} \mathrm{H}_{-}{ }^{15} \mathrm{~N}$ SOFAST-HMQC NMR spectra can only detect $\mathrm{N}-\mathrm{H}$ signals from backbone or from asparagine and glutamine side chains. Thus, these experiments may not reveal changes in the chemical environment of long side chain bearing no $\mathrm{N}-\mathrm{H}$ group. Therefore, for a fair comparison of NMR and MD data, we first compared residues with chemical shift perturbations detected by NMR spectroscopy (top row of Fig. 4) to residues having an $\mathrm{N}-\mathrm{H}$ group distant by less than $5 \AA$ from a calcium ion in our wiCa-noPhos simulations (middle row of Fig. 4). Recovering the observations reported by Feng et al., our NMR data show that the N-H groups which are the most affected by calcium belong to residues His77, Gln79, Asn128, Asn131, Tyr132, Gln133, Asn139, Asn141, Tyr151, and Glu153. In a fairly good agreement, these residues were retrieved in MD simulations, although additional residues contacted by calcium ions, including Glu12, Asp45, Glu58, Glu60, Glu138, and $\operatorname{Arg} 152$, were also identified (middle row of Fig. 4). Moreover, MD simulations revealed many other binding sites, most of which are the negatively charged carboxylate groups of Asp or Glu side chains which cannot be captured by NMR ${ }^{1} \mathrm{H} /{ }^{15} \mathrm{~N}$ chemical shift perturbation measurements (bottom row of Fig. 4). However, we detected a dramatic diminishing of the backbone ${ }^{1} \mathrm{H}^{15} \mathrm{~N}$ resonance intensities in the loop (38-66) upon calcium ad- 
dition (Fig. S3) which was not reported by Feng et al. [11]. We attribute this observation to a change in loop mobility upon calcium binding. To this regard, multiple calcium binding sites were detected within the loop, as demonstrated by MD simulations, and might explain the effect of calcium on the loop dynamics.

In order to visually describe the preferential binding sites of calcium on TCTP, we superimposed the representative structure of the ten most populated clusters of the wiCa-noPhos conformational ensemble, and displayed the ions when at least three calcium were located at the same site (Fig. 5). Combined with previous NMR chemical shift perturbations and MD frequent contacts, this visual inspection allows to identify seven calcium binding sites constituted by residues listed in Fig. 5. The two most occupied calcium binding sites are made up by at least five residues, and the other ones by less than four residues. Interestingly, the TCTP disordered loop is often observed folded towards the core domain in such way that Glu58 and Glu60, which were shown as critical for calcium binding by mutagenesis studies [12], contribute to strengthen the fourth and fifth binding sites made up by residues Gln79, Glu80, and Gln133, confirming the important role of Glu58 and Glu60 in calcium sequestration.

Strikingly, the two most occupied calcium binding regions coincide with the two previously identified patches on the TCTP core domain which are transiently contacted by the disordered loop (Fig. 3). Since the disordered loop is composed of several negatively charged residues, this suggests that the presence of calcium should shift the TCTP conformational ensemble towards structures in which the loop is folded over the calcium ions bound to the core 
domain. This is indeed what can be observed in the plot of the free energy surface of the wiCa-noPhos TCTP as a function of its radius of gyration and the ratio of the distance TCTP1-TCTP2a over the distance TCTP1-TCTP2b (top graph of Fig. 6). When compared to the noCa-noPhos conformational ensemble (Fig. 2), the TCTP intrinsically disordered loop adopts significantly less extended conformations in presence of calcium, the protein radius of gyration of the most populated structures being below $1.7 \mathrm{~nm}$. The presence of calcium induces a more restricted conformational ensemble of the disordered loop, with three major conformations, the first one with TCTP1 in contact with TCTP2a, the second one with TCTP1 close to TCTP2b, and the third one characterized by a proximity of TCTP1 with region 11-12,76-77. In the first two cases, the residues involved in these contacts were identified using distance matrix between the residues 48-58 and 129-151 computed for the ten most populated clusters of TCTP in the presence of calcium. The most significant core-loop contacts were detected for cluster5 and cluster10 (Fig. S4). In the former, hydrophobic interactions were observed between residues Ile48, Gly49, and Ala52 (TCTP1) and residue Pro142 (TCTP2b). In the latter, residues Pro57 and Glu58 (TCTP1) rather make polar contacts with residues Gln133 and Tyr151 (TCTP2a). These two modes of interaction of the disordered loop to the core domain are similar to those previously described for the non-phosphorylated TCTP in the absence of calcium, but are favoured over the detached conformations of the loop by the binding of calcium ions on the surface of the core domain. 


\section{Influence of phosphorylations on TCTP conformational ensemble}

Several cellular and molecular studies indicate that TCTP is involved in the regulation of the dynamics of microtubules and/or microfilaments by binding to tubulin and/or actin [28-31]. This biological activity was shown

to depend on the hierarchical phosphorylation by polo-like kinase Plk1 of the TCTP Ser46 and Ser64 which are located in the intrinsically disordered loop [22, 30-32]. In addition, two pharmacological molecules, sertraline and thioridazine, were shown to bind directly to TCTP and prevent its association to MDM2, but these two compounds were unable to bind TCTP when introducing mutations S46E or S64E which mimic phosphorylated aminoacids [33]. These considerations led us to study the influence of the single phosphorylation pS46 and di-phosphorylation pS46 and pS64 on the TCTP disordered loop conformational ensemble, to gain insight into the role of these post-translational modifications. MD simulations of the modified TCTP, in the same conditions as the non-phosphorylated protein in the absence of calcium, yielded the free energy surfaces displayed in Fig. 6. When compared with Fig. 2, it can be observed that the single phosphorylation pS46 overall induces a slight shift of the TCTP conformational ensemble towards less extended structures among which the disordered loop can fold towards the bottom of the TCTP core domain, but preferentially closer to the TCTP2a segment 129-133 than to the TCTP2b region 138-143 which is more negatively charged. When TCTP is diphosphorylated, its conformational ensemble is also slightly shifted towards less extended structures, but in contrast with the non- and mono-phosphorylated protein, the two phosphate groups reduce the propensity of the disordered loop to bind both the TCTP2a and 
TCTP2b regions and keep it detached from the core domain.

A quantitative analysis of the contacts between residues of the TCTP core domain and its disordered loop confirmed that the contacts made by the region 130-160 with the disordered loop are reduced in number and duration in the di-phosphorylated TCTP with respect to the non-phosphorylated one (Fig. S5). In the case of the mono-phosphorylated pS46 protein, it can be noted that the residues 80-82, 135, and 140 (TCTP2b) are less often contacted by the disordered loop, whereas the segments 71-75 and 129-130 have longer contacts than in the non-phosphorylated TCTP (Fig. S5). Among the ten most populated clusters of the phosphorylated pS46-TCTP, we found again two clusters with the two signatures TCTP1 and TCTP2 in spatial proximity (Fig. S6): cluster4 in which residues Ser53, Glu55, Gly56, and Pro57 make contacts with residues Lys130, Gln133, Phe135, and Tyr151 (TCTP2a), and cluster5 in which residues Asn51 and Ser53 are close to the residue Glu138 (TCTP2b). For the di-phosphorylated protein, we retrieved similar contacts in cluster6 (Asn51 with Glu138) and cluster8 (Asn51, Ala52, and Ser53 with Gln133 and Tyr151) but to a significantly less extent (Fig. S7). It could be noticed that the hydrophobic contacts between TCTP1 and TCTP2b previously observed in the non-phosphorylated protein are not recovered in the phosphorylated TCTP. The phosphorylation-induced weakening of the core-loop contacts might increase the solvent accessibility of some patches at the surface of the TCTP core domain, in particular TCTP2, which could potentially modulate the TCTP binding to other molecular partners.

At the local level, we also examined the impact of the phosphorylation of TCTP residues Ser46 and Ser64 upon the propensity of the disordered loop 
to form secondary structures. As displayed in Fig. S8, the percentages of TCTP structures, in which $\alpha$-helix or $\beta$-strand conformations are observed in the disordered region, remain overall similar (less than 20-25\%) in the four conditions of simulation. Nevertheless, it can be noted that, upon phosphorylation, the helical propensity of segment $44-48$ is decreased, whereas it is slightly increased in region 56-60 with respect to the non-phosphorylated TCTP. More strikingly, TCTP phosphorylation significantly increases the percentage of simulation time in random coil for the phosphorylated serines and adjacent residues when compared to the non-phosphorylated protein. Besides the enhanced electrostatic repulsions between the two highly conserved signatures TCTP1 and TCTP2, serine phosphorylation seems also to favour some local structures of the disordered loop which disadvantage its bridging to the TCTP core domain.

As mentioned above, it was demonstrated that phosphorylations of Ser46 and Ser64 or mimics of these phosphorylations impact the binding of TCTP with tubulin and MDM2 proteins [22, 33] and with TCTP inhibitors [33]. However, as far as we know, the quaternary structures of these TCTPtubulin, TCTP-MDM2, and TCTP-inhibitor complexes are not yet experimentally determined. Some domain mapping studies can suggest the TCTP regions that are involved in binding these proteins but with ambiguity related to this method $[28,33,34]$. In the absence of unambiguous structural information, it is therefore difficult to estimate the implication of TCTP phosphorylations in the interactions with its partners. Nevertheless, we believe that our theoretical results provide some clues that might help to rationalize futur experimental studies about the structures of TCTP-ligand complexes. 
Experimental evidence of a monomer-dimer equilibrium of TCTP in solution

Dimerization of TCTP was previously reported in the literature [35-39]. In this section, we assessed the ability of TCTP to form a concentrationdependent non-covalent dimer through dilution experiments from high (1 mM) to low $(6.25 \mu \mathrm{M})$ concentration of protein using several NMR methods: chemical shift perturbation mapping, diffusion ordered spectroscopy (DOSY), ${ }^{15} \mathrm{~N}$ relaxation as well as their further treatment using Lipari-Szabo formalism via TENSOR2 program. When comparing relaxation parameters over the structured parts at two protein concentrations (50 $\mu \mathrm{M}$ and $500 \mu \mathrm{M})$, we observed lower averaged ${ }^{15} \mathrm{~N} R_{1}$ values at high concentration $\left(<R_{1}>=0.55\right.$ $\left.\pm 0.04 \mathrm{~s}^{-1}\right)$ compared to low concentration $\left(<R_{1}>=0.63 \pm 0.07 \mathrm{~s}^{-1}\right)$. In addition, the averaged ${ }^{15} \mathrm{~N}$ transverse relaxation rate was higher at high concentration $\left(<R_{2}>=21.3 \pm 2.8 \mathrm{~s}^{-1}\right)$ than at low concentration $\left(<R_{2}>\right.$ $=18.4 \pm 2.4 \mathrm{~s}^{-1}$ ) for the TCTP structured parts. Consequently, a higher $\mathrm{R}_{2} / \mathrm{R}_{1}$ ratio was observed at high concentration $\left(<R_{2} / R_{1}>=38.6 \pm 7.6\right)$ compared to low concentration $\left(<R_{2} / R_{1}>=29.0 \pm 6.7\right)$ (Fig. 7A). By using the Lipari-Szabo formalism, we computed an apparent correlation time $\left(\tau_{c}\right)$ that was significantly higher at $500 \mu \mathrm{M}$ concentration $(11.57 \pm 0.06 \mathrm{~ns})$ than at $50 \mu \mathrm{M}$ concentration $(10.10 \pm 0.12 \mathrm{~ns})$, suggesting that a dimer population is enriched when increasing concentration of TCTP. We also performed DOSY experiments in order to measure the translational diffusion coefficient of TCTP as a function of its concentration. We found that the diffusion coefficient gradually decreased from $1.453 \pm 0.008^{*} 10^{-10} \mathrm{~m}^{2} \cdot \mathrm{s}^{-1}$ at low concentration $(100 \mu \mathrm{M})$ to $1.273 \pm 0.003^{*} 10^{-10} \mathrm{~m}^{2} \cdot \mathrm{s}^{-1}$ at high concentration $(1 \mathrm{mM})$ of protein (Fig. S9). This change in TCTP diffusion 
properties could not be attributed to a change in solution viscosity because other solutes, such as the buffer HEPES, had constant diffusion properties over the studied TCTP concentration range (Fig. S9). Hence, this slower translational diffusion at higher concentration is consistent with an increase in the population of TCTP oligomers. The recent native mass analysis of TCTP [40] which revealed the existence of a TCTP dimer together with the limited increase in rotational and translational diffusion properties of TCTP at high concentration suggests that the populated oligomeric form is most likely a dimer in solution.

The ${ }^{1} \mathrm{H}^{15}{ }^{15}$ SOFAST-HMQC spectra for TCTP concentrations from $1 \mathrm{mM}$ to $6.25 \mu \mathrm{M}$ showed clear concentration-dependent chemical shift variations for a subset of residues, including the segment 81-85 which contains the most affected residue (Phe83), the segment 131-135 with residues Asn131, Gln133 and Phe135 showing significant perturbations, and in a lesser extent the segment 149-150. Residues Ile48 and Thr65 in the intrinsically disordered loop were also noticeably disturbed by the dilution. Based on the ${ }^{1} \mathrm{H}$ and ${ }^{15} \mathrm{~N}$ chemical shift variations of the most affected residue Phe83, we could estimate the dissociation constant of the dimer to $1.36 \pm 0.26 \mathrm{mM}$ (Fig. S10). This indicates that TCTP is mostly monomeric at low concentration, the dimer population being lower than $4 \%$ at $50 \mu \mathrm{M}$, whereas this population increases up to $20 \%$ and $30 \%$ at $500 \mu \mathrm{M}$ and at $1 \mathrm{mM}$, respectively. All together, these data strongly evidence the tendency of TCTP to self-associate in vitro in the $\mathrm{mM}$ concentration range. It could be noted that non-covalent dimers of TCTP were also detected in cellulo [35], where a high protein concentration could favor the dimeric form, which might have a biological 
function.

Quaternary structure of TCTP dimer and impact of calcium binding

To get deeper insight into the structure of the dimer formed in solution we analyzed the concentration-dependent NMR chemical shifts information with interfaces derived from several structural models of the dimer. To generate structural models of the TCTP dimer, we considered the representative conformation of the ten most populated clusters from the MD simulations of the non-phosphorylated monomer in the absence of calcium (Fig. 2), and we performed a docking calculation of each of these structures against each of them, using the program PTools and the force field SCORPION. The statistical analysis of the interaction energy of the generated TCTP complexes (Fig. S11) allows to identify five quaternary structures with significantly lower energy than all the other associations. Among this top five, four complexes (dimer1, dimer2, dimer4, and dimer5) exhibit a similar mode of binding in which one of the two TCTP is located at the bottom of the core domain of the other one, these structures differing from each other by the orientation of the first partner (Fig. 8). In order to determine which of these four complexes is the most probable, we computed the number of contacts per residue between the two TCTP of each complex and compared these contact profiles with the NMR chemical shift perturbations upon protein dilution (Fig. 9). This analysis shows that dimer1 and dimer2 have contact profiles in good agreement with NMR chemical shift perturbations, indicating that they are among the most probable structures of the TCTP dimer, but without ruling out dimer5 whose contact profile remains fairly consistent with NMR data. All the more so dimer5 is interestingly the most symmetric complex 
among the top five and its dimerization interface is highly similar to that observed in the crystallographic structures solved by Susini et al. for the human wild type TCTP [24] (Fig. 8), but also for the human E12V mutant [41] and the murine TCTP [42], which might reveal that this interface plays a major role in TCTP crystallisation. It could be noted that, in these three most probable complexes, one of the two partners is either cluster6 or cluster1 which both have the disordered loop in a rather extended conformation, with a short $\alpha$-helix at segment 59-63. In the modelled dimer1, dimer2, and dimer5, this transient helix is in contact with the $\alpha$-hairpin of the interacting partner, contributing to stabilize the TCTP dimer. This latter remark is in line with far-UV CD experiments which indicate a gain of $\alpha$-helical content upon hemin-induced TCTP dimerization [39].

Strikingly, the interface of TCTP dimerization partially overlaps several calcium binding sites previously identified (site 2, 4, and 5 of Fig. 5), suggesting that calcium ions could hinder protein self-association. To verify this hypothesis, we compared the TCTP ${ }^{1} \mathrm{H}_{-}{ }^{15} \mathrm{~N}$ HSQC spectra at low $(50 \mu \mathrm{M})$ or medium $(500 \mu \mathrm{M})$ protein concentration, without or with $50 \mathrm{mM}$ calcium ions. More specifically, we closely examined the positions of the ${ }^{1} \mathrm{H}-{ }^{15} \mathrm{~N}$ cross-peaks of residues Phe83 and Lys85 which were previously shown to be sensitive to dimerization. As shown in Fig. 10, an important shift in crosspeak positions of these residues can be noticed between low and medium protein concentration, in the absence of calcium (top panels). In contrast, in the presence of calcium, the cross-peak positions of Phe83 and Lys85 do not depend on the protein concentration (in this concentration range) and are typical of monomeric TCTP (bottom panels). Taken together, this suggests 
that calcium prevents the formation of TCTP dimer. To confirm this, we compared ${ }^{15} \mathrm{~N}$ relaxation experiments collected at low $(50 \mu \mathrm{M})$ and medium $(500 \mu \mathrm{M})$ TCTP concentration in the absence or in the presence of $50 \mathrm{mM}$ calcium (Fig. 7). In the presence of calcium, the averaged ${ }^{15} \mathrm{~N} \mathrm{R}_{2} / \mathrm{R}_{1}$ ratio over the structured regions of TCTP at medium $(500 \mu \mathrm{M})$ concentration $\left(<R_{2} / R_{1}>=31.0 \pm 6.9\right)$ was close to the value observed for the protein at lower $(50 \mu \mathrm{M})$ concentration $\left(<R_{2} / R_{1}>=29.1 \pm 6.7\right)$. This turned into similar apparent correlation times at $500 \mu \mathrm{M}\left(\tau_{c}=10.62 \pm 0.03 \mathrm{~ns}\right)$ and at $50 \mu \mathrm{M}\left(\tau_{c}=10.10 \pm 0.12 \mathrm{~ns}\right)$ for TCTP in the presence of calcium. Moreover, we observed a significant increase in the translational coefficient diffusion of $\operatorname{TCTP}(500 \mu \mathrm{M})$ from $\mathrm{D}=1.320 \pm 0.004^{*} 10^{-10} \mathrm{~m}^{2} . \mathrm{s}^{-1}$ to $\mathrm{D}=1.370 \pm$ $0.003^{*} 10^{-10} \mathrm{~m}^{2} . \mathrm{s}^{-1}$ when adding calcium ions. All together, these data confirm that the presence of calcium ions prevents the TCTP dimerization at medium protein concentration. Our results are in agreement with those reported by Lucas et al. which demonstrated that the hemin-induced dimeric form of TCTP is destabilized by the addition of calcium ions [39]. We propose that in both situations (in the presence or absence of hemin), calcium ions favor the TCTP monomeric form by a similar mechanism which involves calcium-induced increased interactions between the disordered loop and the core domain. The competitive binding of the loop to the TCTP dimerization interface may contribute to disfavor its self-association, thus exemplifying the potential role of the disordered loop in regulating the TCTP protein-protein interactions. 


\section{Conclusion}

The role of the intrinsically disordered region in TCTP biological activities was poorly studied so far, partly because its conformations were not exhaustively characterized. Yet, this long loop should probably play an im-

portant role since it contains a highly conserved amino-acid signature of TCTP. In the present study, we combined NMR experiments and MD simulations to better characterize its conformational ensemble and investigate its potential role in TCTP functions. We showed that the non-phosphorylated TCTP disordered loop can adopt extended conformations detached from the core domain, but can also fold towards the latter in such way that the protein first signature segment (TCTP1) directly binds to one of the two extreme parts of its second signature region (TCTP2). These long-range transient interactions between TCTP1 and TCTP2 might reveal an underlying mechanism connecting the two signatures. Such mechanism, which still needs to be elucidated, might be a driving force that contributes to the signature sequence conservation. When the two Ser46 and Ser64 of the disordered loop are phosphorylated, the TCTP conformations in which the loop is attached to the core domain are less populated than in the non-phosphorylated protein. In contrast, the presence of calcium ions induces a significant shift of the TCTP conformational ensemble towards structures in which the disordered loop folds against the core domain and contributes to the binding of calcium ions on TCTP surface. In the protein dimers, the disordered loops are pulled away from the core domains to allow TCTP self-association. They also contribute in the stabilization of the protein-protein interface by interacting with the partner $\alpha$-hairpin. All together, we propose that the TCTP intrinsically 
disordered region could play an important role in participating to the interactions with other proteins and non-peptidic ligands, in remodelling the core domain surface, and modulating its accessibility to its partners in response to a variety of cellular conditions, in particularly the presence of calcium ions, or to chemical modifications, such as phosphorylations. We conclude that the TCTP disordered region conformational dynamics must be carefully taken into account when using TCTP structure as a biomolecular target for drug development, particularly for in silico screening.

\section{Materials and Methods}

Expression and purification of the human TCTP

The open reading frame of human TCTP was cloned into pET-M11 vector. A His 6 tag followed by a TEV protease cleavage site was inserted at the N-terminus of TCTP (His-TCTP). ${ }^{15} \mathrm{~N}$ and ${ }^{15} \mathrm{~N}-{ }^{13} \mathrm{C}$ labeled TCTP were expressed in E. coli BL21 star (DE3) (Life Technologies) grown in M9 minimal medium containing ${ }^{15} \mathrm{~N}$-labeled $\mathrm{NH}_{4} \mathrm{Cl}(1 \mathrm{~g} / \mathrm{L})$ and ${ }^{12} \mathrm{C}-\alpha$-D-glucose $(4 \mathrm{~g} / \mathrm{L})$ or ${ }^{15} \mathrm{~N}$-labeled $\mathrm{NH}_{4} \mathrm{Cl}(1 \mathrm{~g} / \mathrm{L})$ and ${ }^{13} \mathrm{C}$-labeled $\alpha$-D-glucose $(4 \mathrm{~g} / \mathrm{L})$, respectively. Overexpression was induced when $\mathrm{OD}_{600}$ reached 0.9 by addition of $0.2 \mathrm{mM}$ isopropyl- $\beta$,D-thiogalactopyranoside (IPTG) and lasted 20 hours at $25{ }^{\circ} \mathrm{C}$. Cell pellets were resuspended at 1:10 w/v in lysis buffer $(50 \mathrm{mM}$ Tris pH 8, $300 \mathrm{mM} \mathrm{NaCl}, 10 \mathrm{mM}$ imidazole, $2 \mathrm{mM}$ DTT) supplemented with $0.3 \mathrm{mg} / \mathrm{ml}$ lysozyme and protease inhibitor cocktail (Roche) and lysed using a French press system (1500 bars, 3 cycles). After centrifugation (125000 g, $60 \mathrm{~min}$ ), supernatant was loaded on a Histrap Fast Flow crude column (GE Healthcare life sciences) and His-TCTP was eluted with $150 \mathrm{mM}$ imidazole. 
The N-Terminal $\mathrm{His}_{6}$ tag was removed by digesting the His-TCTP protein with home-made His-TEV protease $(1: 50 \mathrm{w} / \mathrm{w}$ ratio) and simultaneously dialyzed against imidazole-free buffer (50 mM Tris pH 8, $300 \mathrm{mM} \mathrm{NaCl,} 2 \mathrm{mM}$ DTT) during 15 hours at $34^{\circ} \mathrm{C}$. TCTP was separated from His-TCTP and His-TEV by passing the digest products on a Histrap Fast Flow crude column (GE Healthcare life sciences) and further purified on a Superdex 75 10/300 GL column (GE Healthcare life sciences) equilibrated with storing buffer (50 mM HEPES pH 7.4, $150 \mathrm{mM} \mathrm{NaCl,} 2$ mM TCEP, 0.5 mM EDTA). Human TCTP was concentrated at $2 \mathrm{mM}$ and stored at $-80{ }^{\circ} \mathrm{C}$.

\section{NMR spectroscopy}

NMR spectroscopy measurements were performed at $298 \mathrm{~K}$ in the following buffer: 50 mM HEPES pH 7.4, 150 mM NaCl, 2 mM TCEP. Measurements were done using Bruker AVIII $950 \mathrm{MHz}$ and $800 \mathrm{MHz}$ spectrometers equipped with a TCI cryoprobe. Sequence-specific backbone assignment of human TCTP was achieved using a classical approach [43] and on the basis of the published assignment [11]. All NMR data, except ${ }^{15} \mathrm{~N}$ relaxation measurements, were processed with Topspin 3.5 (Bruker) and analyzed with CCPNMR software [44]. Coupling constants ${ }^{3} J_{H_{N}-H \alpha}$ were measured from a 3D HNHA experiment [45]. The intensities of the diagonal $\left(S_{\text {diag }}\right)$ and off-diagonal $\left(S_{\text {cross }}\right)$ cross-peaks were extracted and coupling constants were computed according to the following equation: $S_{\text {cross }} / S_{\text {diag }}=$ $-\tan ^{2}\left(2 \pi J_{H H} \zeta\right)$ with $\zeta=13.05 \mathrm{~ms}$. For calcium binding studies and TCTP dilution experiments, combined ${ }^{1} \mathrm{H}^{15} \mathrm{~N}$ chemical shift perturbations $\left(\Delta \delta_{\text {comb }}\right)$ were calculated according to the equation $\Delta \delta_{\text {comb }}=\left(\Delta \delta^{1} H+0.14 \Delta \delta^{15} N\right)^{1 / 2}$, where $\Delta \delta^{1} H$ and $\Delta \delta^{15} N$ are the chemical shift perturbations (in ppm) for 
${ }^{1} \mathrm{H}$ and ${ }^{15} \mathrm{~N}$ resonances, respectively. Such calculation was done by recording series of ${ }^{1} \mathrm{H}-{ }^{15} \mathrm{~N}$ SOFAST-HMQC spectra [46]. TCTP self-dissociation constant $K_{d}$ was estimated by fitting the ${ }^{1} \mathrm{H}$ and ${ }^{15} \mathrm{~N}$ chemical shift perturbation of Phe83 cross-peak upon protein dilution from $1 \mathrm{mM}$ to $6.25 \mu \mathrm{M}$ with the following equation: $\Delta \delta^{A} X=\Delta \delta^{A} X_{0}+\Delta \delta^{A} X_{\max } * F_{d}$, where ${ }^{A} X$ denotes either ${ }^{1} \mathrm{H}$ or ${ }^{15} \mathrm{~N}$ nucleus, and $F_{d}$ the fraction of dimer which is related to $K_{d}$ and TCTP total concentration according to the following equation:

$$
F_{d}=\frac{4 *[T C T P]+K_{d}-\sqrt{8 *[T C T P] * K_{d}+K_{d}^{2}}}{4 *[T C T P]-K_{d}+\sqrt{8 *[T C T P] * K_{d}+K_{d}^{2}}}
$$

${ }^{15} N$ relaxation measurement

Relaxation experiments were performed using a Bruker AVIII HD $950 \mathrm{MHz}$ spectrometer at $298 \mathrm{~K}$ with a ${ }^{15}$ N-labeled TCTP at low $(50 \mu \mathrm{M})$ and medium $(500 \mu \mathrm{M})$ concentration, in the absence and in the presence of $\mathrm{CaCl}_{2}(50 \mathrm{mM})$, and processed using NMRPipe software [47]. The ${ }^{15} \mathrm{~N}$ relaxation experiments were recorded in interleaved pseudo-3D fashion to attenuate the effects of sample and/or conditions changes during the collection time. The ${ }^{15} \mathrm{~N} \mathrm{R}_{1}$ values were determined from series of $2 \mathrm{D}^{1} \mathrm{H}^{15} \mathrm{~N}$ correlation spectra recorded with the following delays (ms): 10, 3000, 200, 2500, 400, 2000, 600, 1500, 800, and 1000 (in this order). The ${ }^{15} \mathrm{~N} \mathrm{R}_{2}$ values were determined with different relaxation delays (ms): 17, 170, 34, 102, 0, 51, 85, and 68 (in this order). $\left\{{ }^{1} \mathrm{H}\right\}-{ }^{15} \mathrm{~N}$ heteronuclear NOE experiments were recorded using one reference and one proton-saturated $2 \mathrm{D}^{1} \mathrm{H}_{-}{ }^{15} \mathrm{~N}$ correlation experiment. ${ }^{15} \mathrm{~N} \mathrm{R} \mathrm{R}_{1}, \mathrm{R}_{2}$, and $\left\{{ }^{1} \mathrm{H}\right\}-{ }^{15} \mathrm{~N}$ heteronuclear NOE were recorded with an interscan delay of 5, 3.5, and $4.8 \mathrm{~s}$, respectively. Intensities from $\mathrm{R}_{1}, \mathrm{R}_{2}$, and $\left\{{ }^{1} \mathrm{H}\right\}^{-15} \mathrm{~N}$ heteronuclear NOE experiments were extracted using the nlinLS routine in NMRPipe and 
$R_{1}$ and $R_{2}$ values were obtained by fitting intensities with a two-parameter exponential model with the modelXY tool in NMRPipe. Further analysis of ${ }^{15} \mathrm{~N}$ relaxation parameters and $\left\{{ }^{1} \mathrm{H}\right\}^{-15} \mathrm{~N}$ heteronuclear NOE in terms of rotational diffusion tensor was achieved by using TENSOR2 program [48].

\section{Molecular dynamics simulation}

The TCTP conformational ensembles were explored under four conditions: no calcium and no phosphorylation (noCa-noPhos), with calcium and no phosphorylation (wiCa-noPhos), no calcium and with phosphorylated Ser46 (noCa-pS46), and lastly no calcium and with both Ser46 and Ser64 phosphorylated (noCa-diPhos). For each condition, the TCTP conformational space was sampled using 20 independent simulations starting from the 20 NMR structures of the PDB file 2HR9 [11]. The phosphorylations of residues Ser46 and Ser64 were done using the Structure Editing tools of the UCSF Chimera package [49]. All initial conformations were solvated in a triclinic box type with a minimum distance of $1.4 \mathrm{~nm}$ between the solute and the box sides. The total charges were neutralized by adding sufficient sodium and chloride ions to reach the salt concentration of $100 \mathrm{mM}$. In simulations with calcium, ten $\mathrm{Ca}^{2+}$ ions were randomly placed in the solvent bulk, corresponding to a calcium concentration of $30 \mathrm{mM}$ in average.

Molecular dynamics (MD) simulations were performed using the GROMACS4.5.5 software $[50,51]$, at the all-atom level in explicit solvent. We used for this study the AMBER-99SB-ILDN force field [52] and the TIP3P water model [53]. The non-bonded interactions were treated using the smooth PME method [54] for the electrostatic terms and a cutoff distance of $1.2 \mathrm{~nm}$ for the van der Waals potentials. The covalent bond lengths were kept con- 
stant using the LINCS [55] and SETTLE [56] procedures for the protein and water molecules, respectively. A leap-frog algorithm was used to integrate the equations of motion with a 2 fs time step. Each MD simulation was run for $200 \mathrm{~ns}$ in the NPT ensemble, at $T=310 \mathrm{~K}$ and $P=1 \mathrm{bar}$, using the Nose-Hoover and Parrinello-Rahman algorithms [57-59], with the time coupling constants $\tau_{T}=0.5 \mathrm{ps}$ and $\tau_{P}=2.5 \mathrm{ps}$.

Molecule coordinates were saved every 20 ps for subsequent analysis, using mainly the tools implemented in the GROMACS package. Notably, we made principal component analyses of the protein conformational ensembles using g_covar and projected the trajectories onto the first two eigenvectors with g_anaeig, yielding for the four TCTP ensembles the reduced free energy surfaces displayed in Fig. S12. These analyses allowed to identify and objectively separate the most populated sub-ensembles or clusters of the TCTP conformations. However, it is not straightforward to interpret the eigenvectors of a principal component analysis in terms of geometrical quantities. Moreover, the first two principal axis are not necessarily identical for the four TCTP conformational ensembles (noCa-noPhos, wiCa-noPhos, noCa-pS46, and noCa-diPhos), which makes comparisons between the four systems very difficult. For these reasons, we chose to analyse the TCTP conformational ensembles in terms of the more intuitive radius of gyration and distances between the two signatures TCTP1 and TCTP2. More specifically, we used the ratio of the distance TCTP1-TCTP2a over TCTP1-TCTP2b in order to highlight the propensity of the TCTP loop to bind either one or another area of the TCTP core domain.

Theoretical chemical shifts were calculated using the SHIFTS program [60, 
61]. Multiple secondary chemical shifts were subsequently combined into a single secondary structure propensity (SSP) score following the Marsh et al. approach [27]. Coupling constants ${ }^{3} J_{H_{N}-H_{\alpha}}$ were calculated from the dihedral angles $\phi$ and $\psi$ using the Karplus equation [62] and the Vuister and Bax empirical parameters [45].

Bootstrap analysis of the TCTP conformational ensembles

In order to assess the reliability of our theoretical results, particularly the significance of the differences between the four TCTP conformational ensembles (noCa-noPhos, wiCa-noPhos, noCa-pS46, and noCa-diPhos), a bootstrap analysis was performed on the protein radius of gyration and the distance between the two signatures TCTP1 and TCTP2. The bootstrapping consisted in reconstructing $4 \mu$ s long trajectories with twenty short 200 ns trajectories randomly selected with replacement among the performed twenty MD simulations. This resampling was iterated 1000 times and the statistics on the TCTP radius of gyration and the TCTP1-TCTP2 distance (averages and standard deviations) were calculated over the 1000 reconstructed trajectories.

The bootstrap analysis yielded the probability distributions displayed in Fig. 11. It is observed that the distributions of both the radius of gyration and the TCTP1-TCTP2 distance are clearly shifted toward lower values in the presence of calcium when compared to simulations without calcium. The error bars show that this difference is significant and reliable. In contrast, the differences between the conformational ensembles of the phosphorylated and non-phosphorylated proteins are less marked. Nevertheless, in the distributions of the TCTP1-TCTP2 distance, one can detect a higher probability of 
distances below $0.5 \mathrm{~nm}$ for the pS46 TCTP and a more subtle higher probability of distances between 0.5 and $1.0 \mathrm{~nm}$ for the di-phosphorylated protein than for the non-phosphorylated one.

\section{Protein-protein docking}

Structures of the TCTP dimer were generated using the molecular modeling library PTools [63]. This toolbox enables to perform protein-protein docking at a coarse-grained level by multiple energy minimizations without any bias, using possibly various force fields. We used in this study the coarsegrained force field SCORPION which was able to successfully reproduce the quaternary structure of several protein-protein complexes, starting from the bound conformation of the two partners $[64,65]$. PTools performs systematic

rigid-body docking, starting from initial regular positions and orientations of the ligand around the receptor surface, at a distance slightly larger than its largest dimension. The docking procedure consists in minimizing the interaction energy between the two partners, using the ligand six translational and rotational degrees of freedom. The minimized complex conformations were finally clustered by similarity and ranked according to their interaction energies.

\section{Acknowledgements}

This work was supported by the LabEx LERMIT (Grant ANR-10-LABX33) and by the project IDI 2016 funded by the IDEX Paris-Saclay (Grant ANR-11-IDEX-0003-02). Financial support from the TGIR-RMN-THC FR3050 CNRS for conducting the research is gratefully acknowledged. Simulations were performed using HPC resources from GENCI-CINES (Grant c2015077390). 
T.H.D. is also thankful for the support from the french Ministry of Foreign Affairs (Grant Bio-Asia-2015 34058UC). Lastly, the authors are warmly grateful to François Bontems for sharing the equation relating the fraction of dimer to the self-dissociation constant.

\section{Conflict of interests}

The authors declare no conflict of interests regarding the publication of this article. 


\section{Figures}

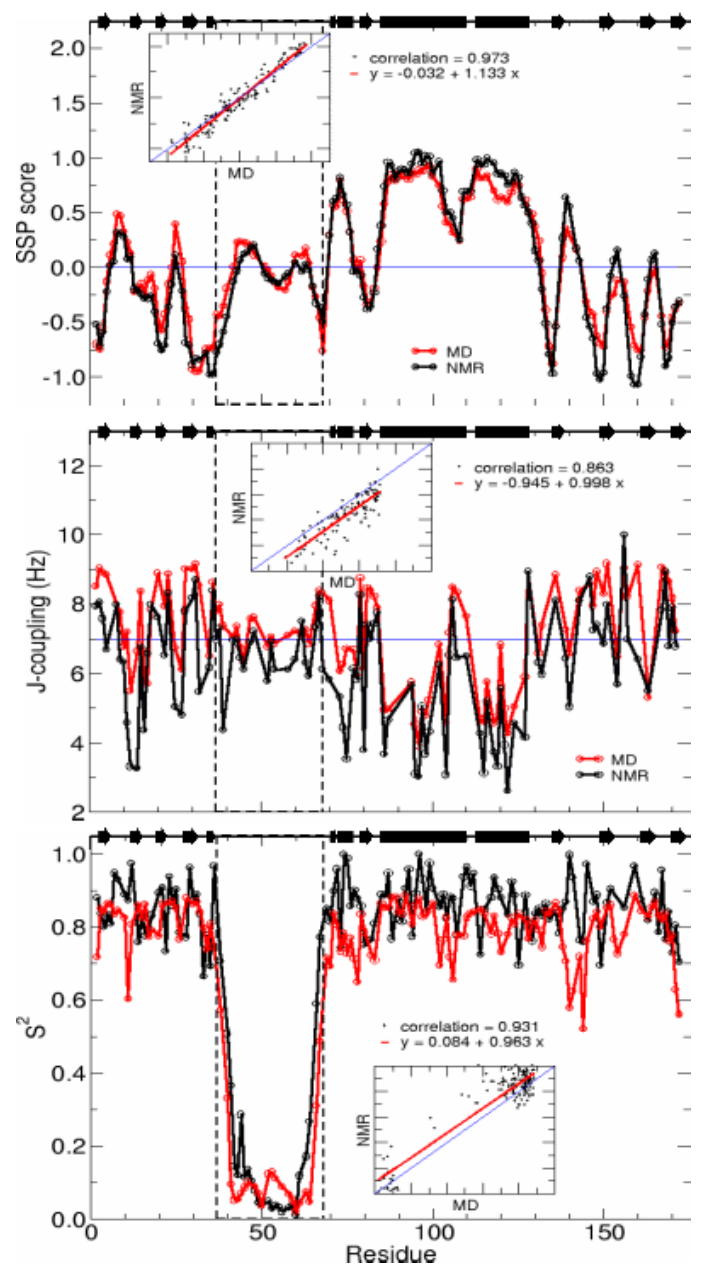

Figure 1: Comparison between NMR (black) and noCa-noPhos MD (red) SSP scores calculated from $\mathrm{C} \alpha, \mathrm{C} \beta$ and $\mathrm{H} \alpha$ chemical shifts (top), coupling constants ${ }^{3} \mathrm{~J}_{H_{N}-H_{\alpha}}$ (middle), and $\mathrm{N}-\mathrm{H}$ bond order parameters $\mathrm{S}^{2}$ as a function of residue number. NMR experiments were done at $50 \mu \mathrm{M}$ TCTP concentration in the buffer HEPES $50 \mathrm{mM}$ pH $7.4150 \mathrm{mM}$ $\mathrm{NaCl} 2 \mathrm{mM}$ TCEP at $298 \mathrm{~K}$. 


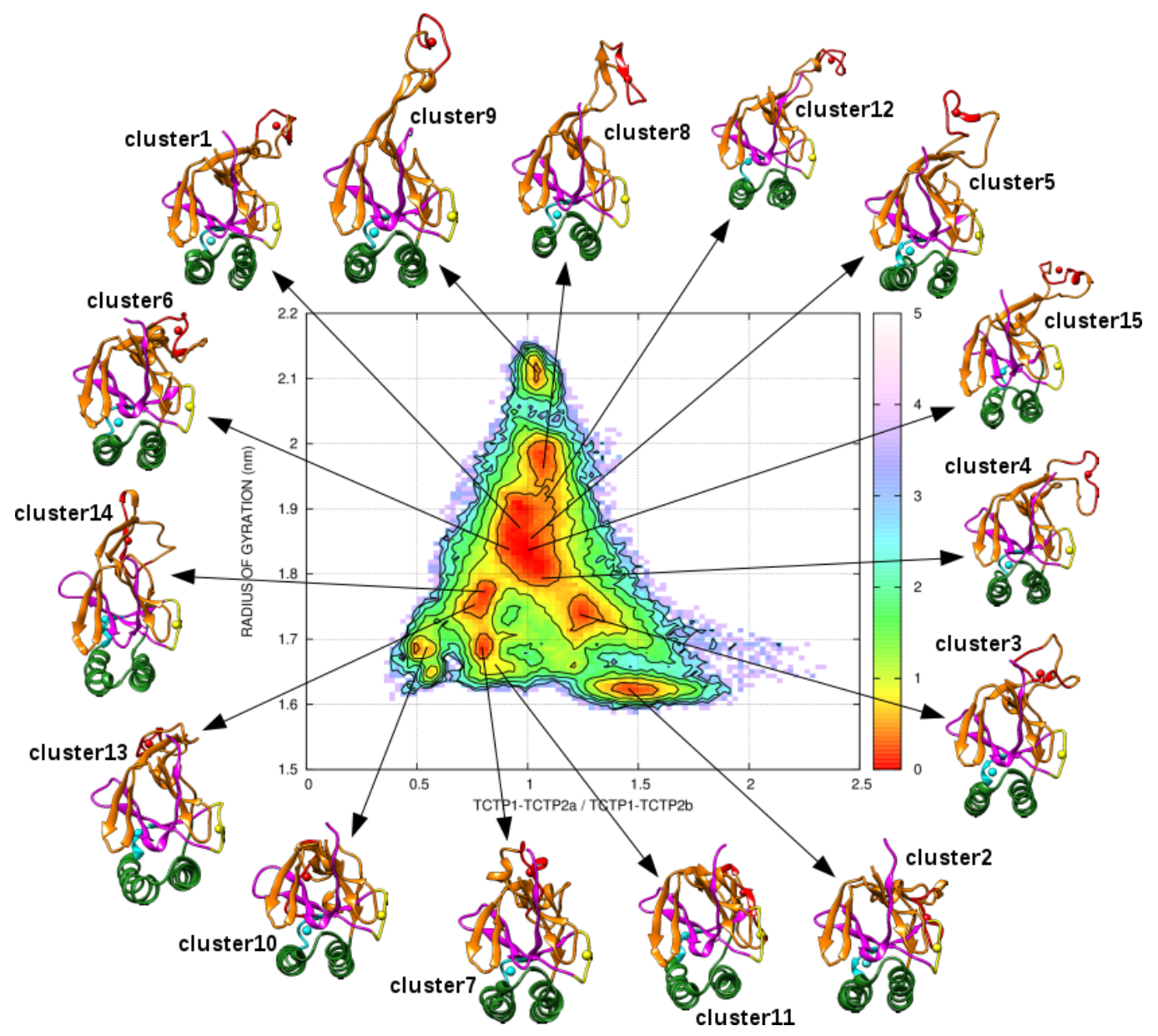

Figure 2: Free energy surface (kcal/mol) of the non-phosphorylated TCTP in the absence of calcium calculated as a function of its radius of gyration and the ratio of the distance between the centers of mass of the signature TCTP1 (region 48-58, red ball) and of residues 129-133,150-151 (TCTP2a, cyan ball) to the distance between the centers of mass of TCTP1 and of residues 138-143 (TCTP2b, yellow ball). The representative conformation of the 15 most populated clusters are displayed in top view. 


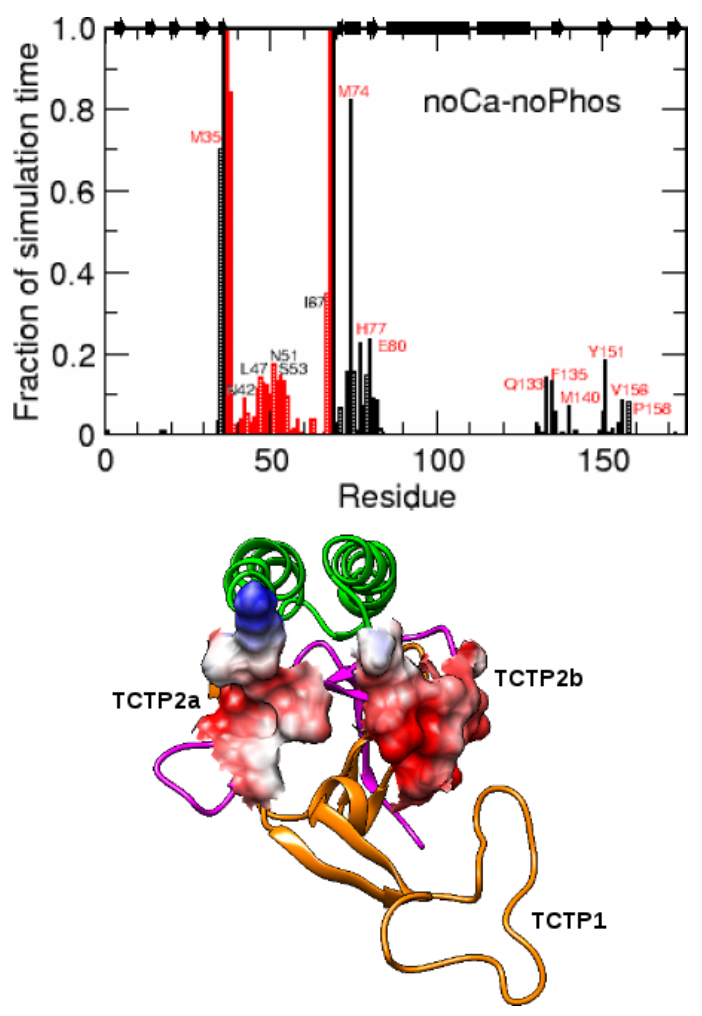

Figure 3: Top: Fraction of the simulation time for which each residue of the TCTP core domain (black), or of the disordered loop (red), is contacted (distance $<3 \AA$ ) by the long loop or the core domain, respectively. Bottom: Bottom view of the two areas on the TCTP core domain which are the most affected by the binding of its intrinsically disordered loop. The first patch is composed of segments 77-82 and 138-141 (TCTP2b). The second one is composed of residues 130-133 and 150-151 (TCTP2a). These surfaces are colored as a function of their local charge density (red: negative, white: neutral, and blue: positive). The loop structure displayed here is only one of many conformations of the TCTP ensemble. 


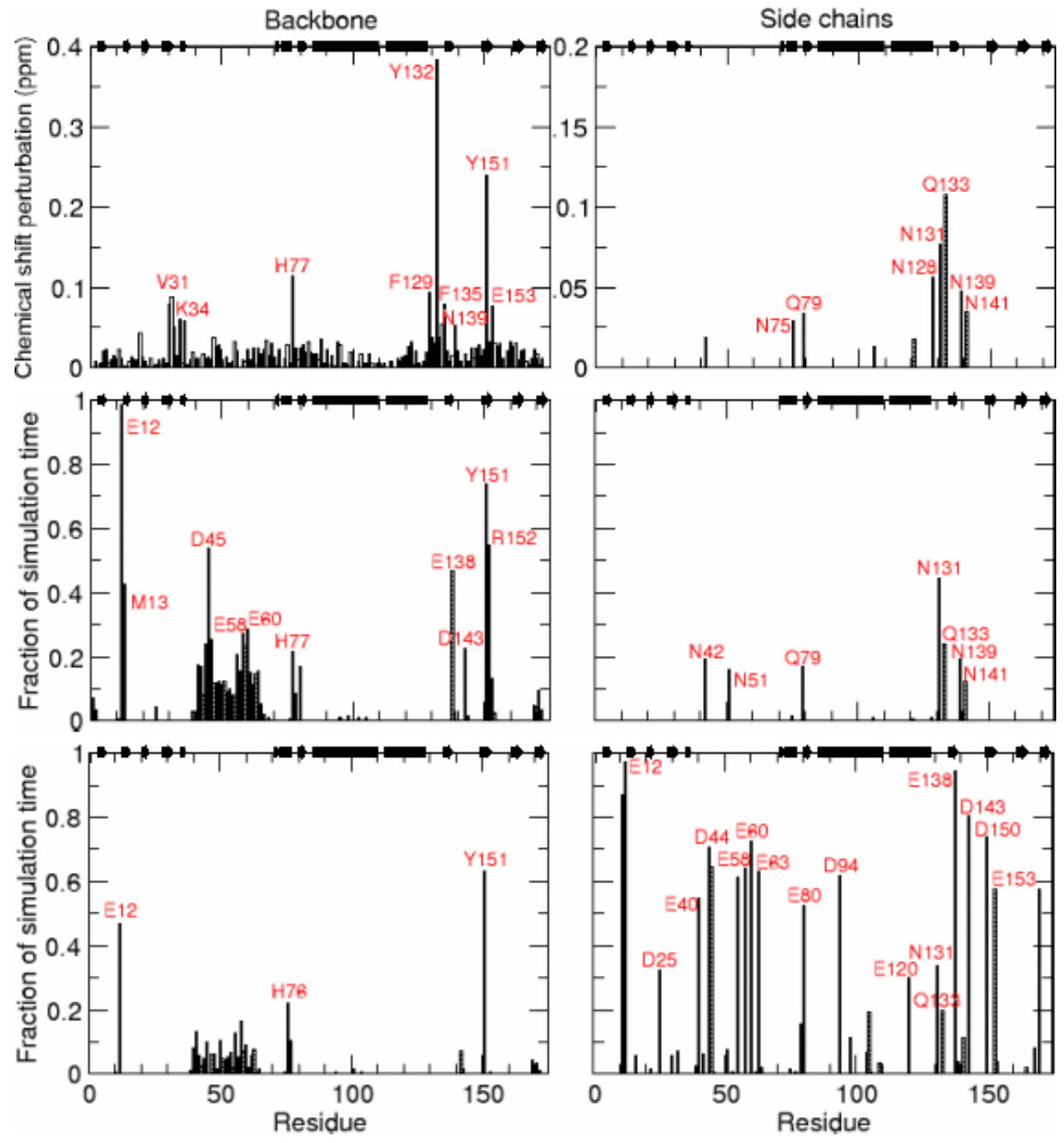

Figure 4: Top: NMR ${ }^{1} \mathrm{H}^{-15} \mathrm{~N}$ chemical shift perturbations of TCTP backbone (left) and Asn or Gln side chain (right) amide protons induced by the presence of $20 \mathrm{mM}$ calcium ions. Middle: Fraction of simulation time for which calcium ions are distant by less than $5 \AA$ to the TCTP backbone (left) and Asn or Gln side chain (right) amide protons. Bottom: Fraction of simulation time for which calcium ions are distant by less than $3 \AA$ to the TCTP backbone (left) and side chains (right) atoms. 


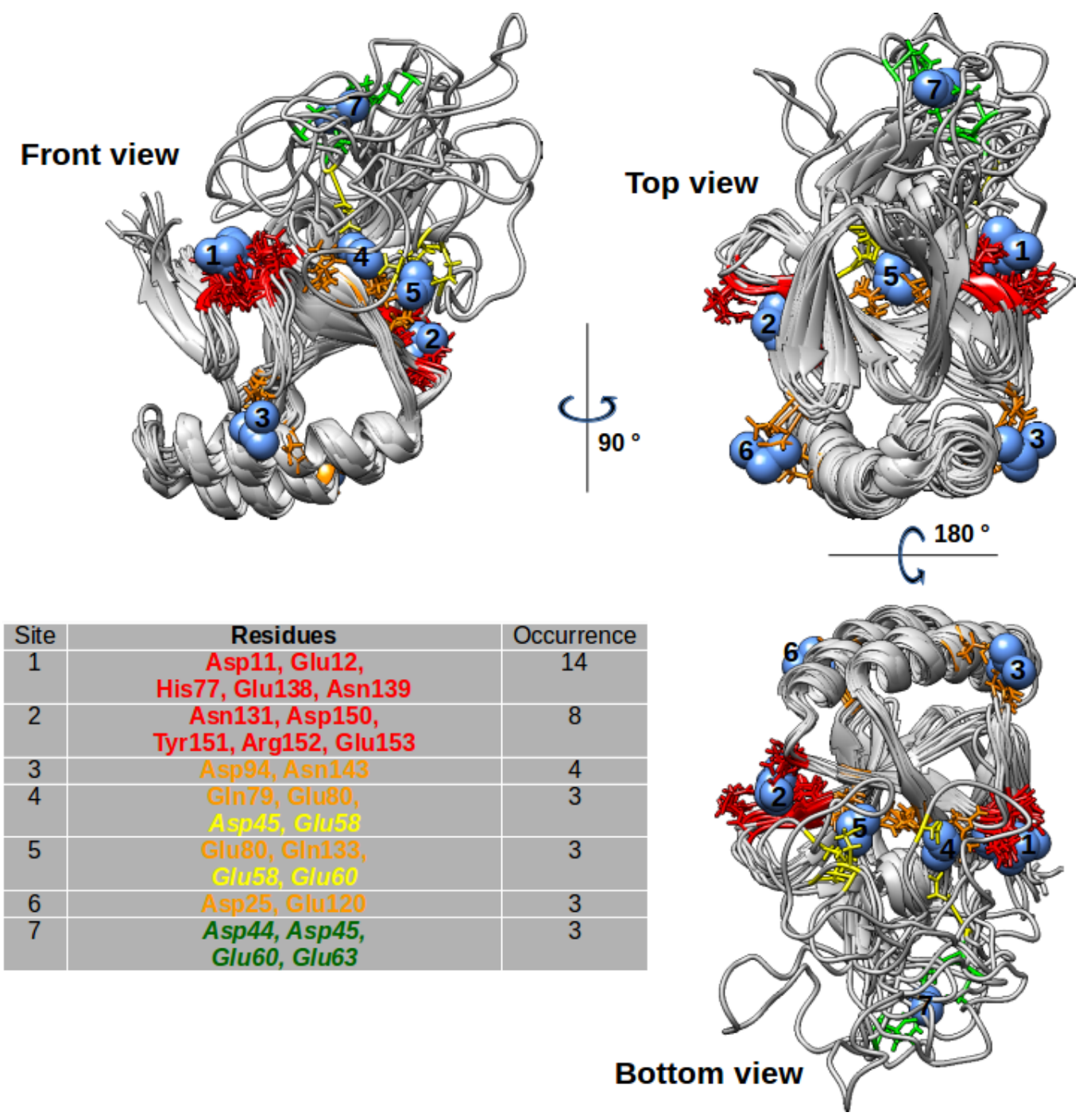

Figure 5: Front, top, and bottom views of the seven preferential binding sites of calcium on TCTP. These illustrations were obtained by superimposing the representative structures of the 10 most populated clusters of the wiCa-noPhos conformational ensemble. The bottom left table lists the residues that compose each binding site and the total number of calcium found there in these 10 most populated clusters (occurrence). 

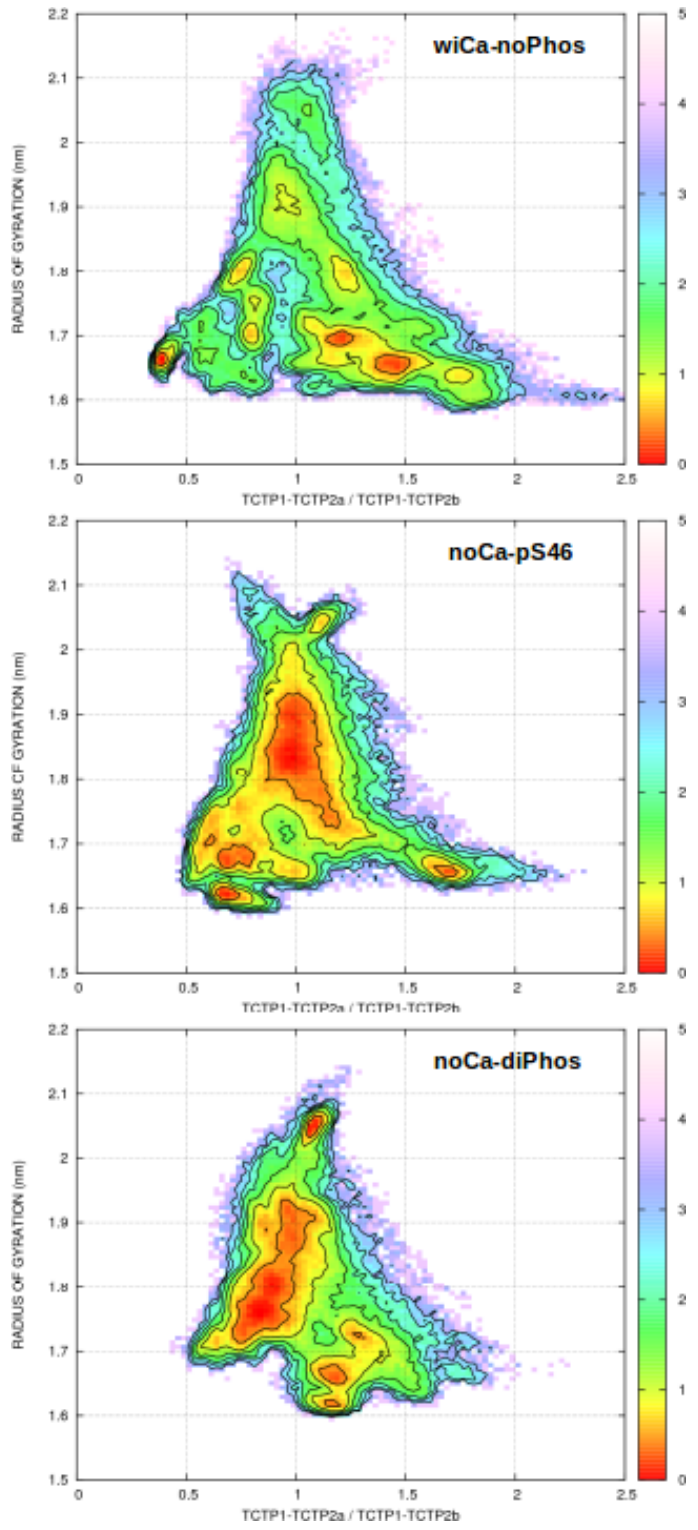

Figure 6: Same free energy surface (kcal/mol) as in Fig. 2 but for the non-phosphorylated TCTP in the presence of calcium (top), the phosphorylated pS46 (middle) and the diphosphorylated pS46 and pS64 protein in the absence of calcium (bottom). 

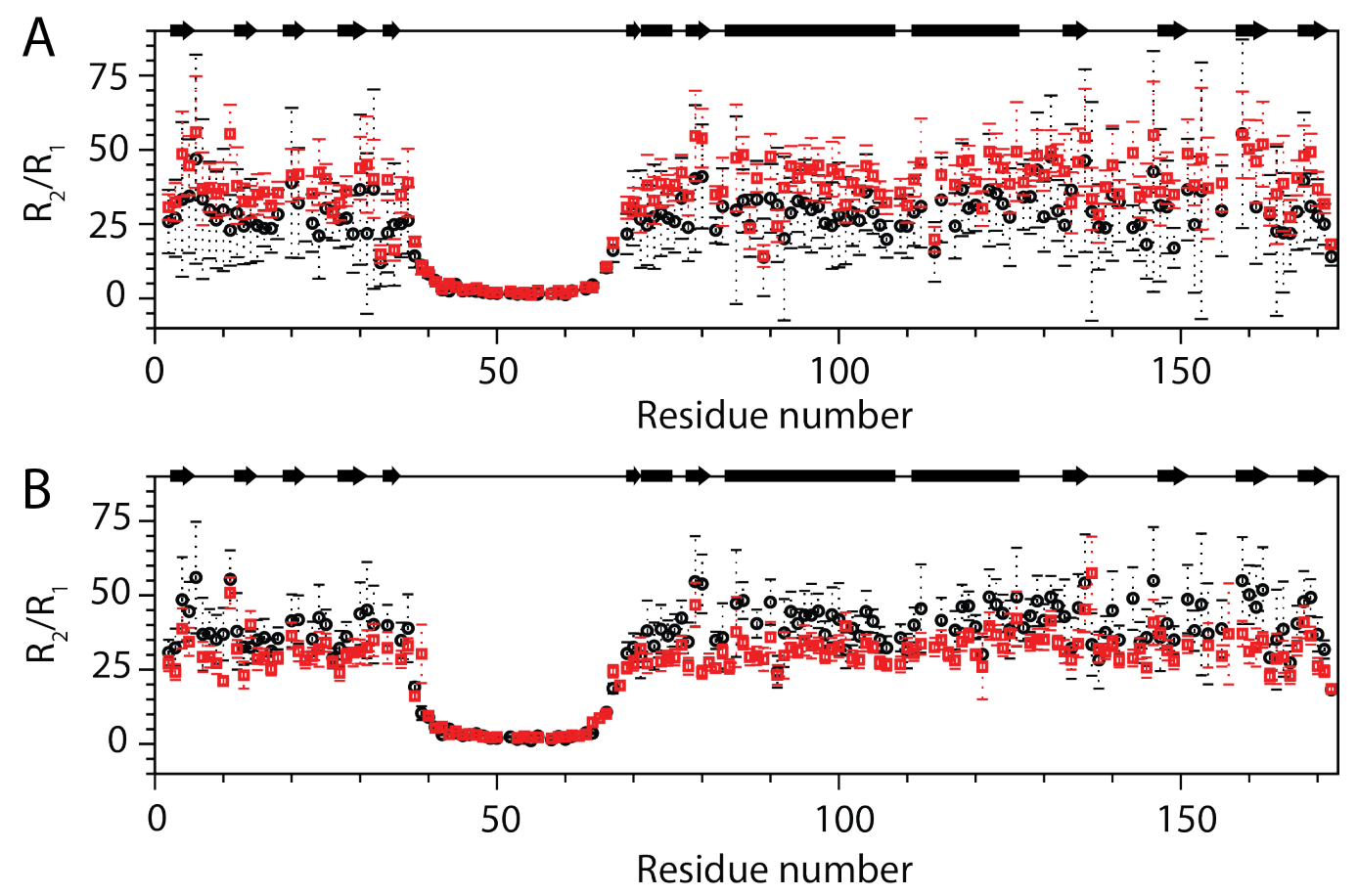

Figure 7: Comparison of ${ }^{15} \mathrm{~N} \mathrm{R}_{2} / \mathrm{R}_{1}$ ratio between low (50 $\mu \mathrm{M}$, black) and medium (500 $\mu \mathrm{M}$, red) concentrations of TCTP in the absence of calcium (A), and for medium concentration of TCTP $(500 \mu \mathrm{M})$ in the absence (black) or in the presence of $50 \mathrm{mM}$ calcium ions (red) (B). 

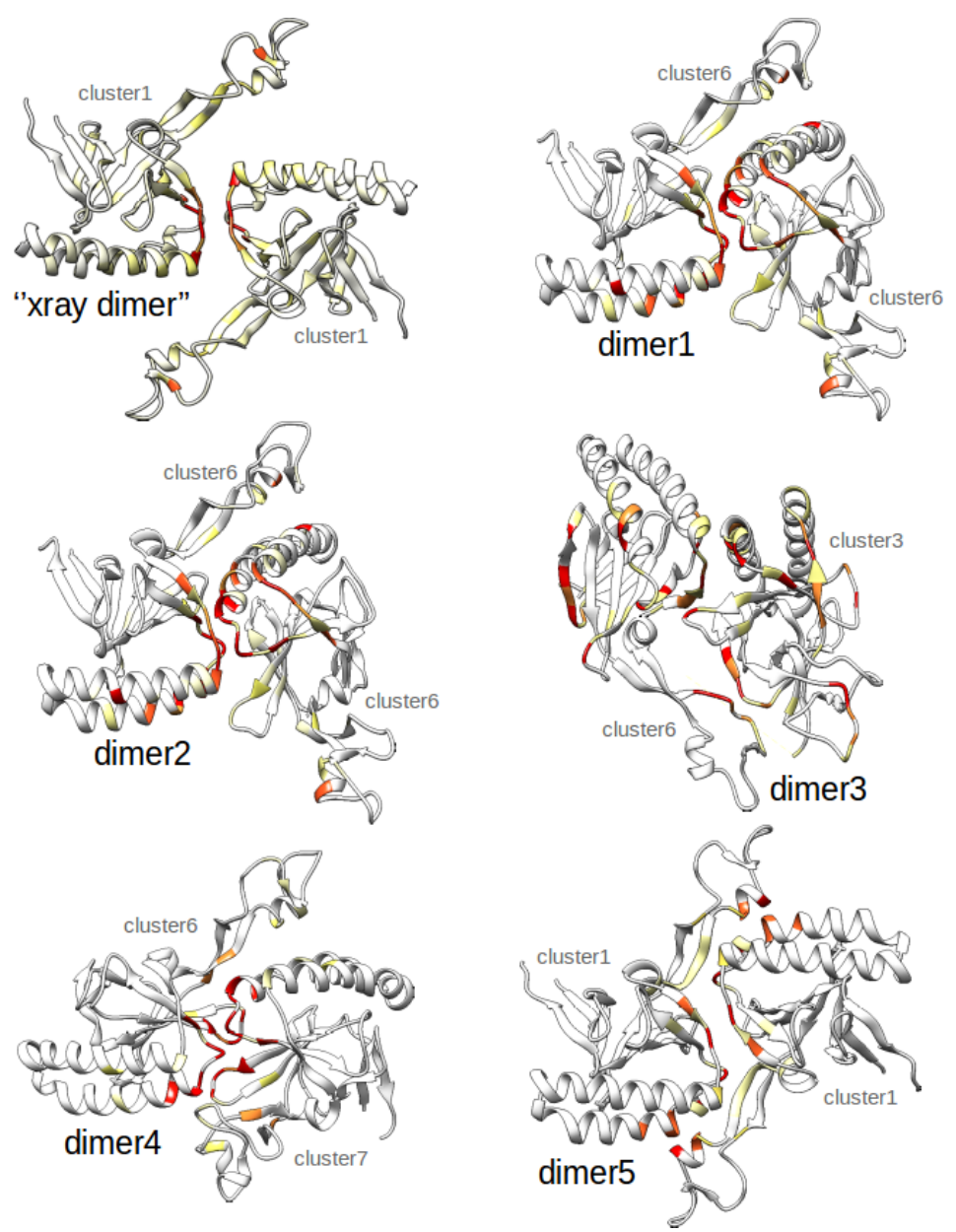

Figure 8: Top left: Structural model of a TCTP dimer built by superimposing the cluster1 on the dimer observed between chains A and D of the PDB structure 1YZ1 [24]. Top right, middle, and bottom: Structures of the five lowest energy TCTP dimers obtained by docking calculations. The residues which exhibit large, medium, or low chemical shift perturbation in NMR dilution experiment or number of contacts in docked complexes are highlighted in red, orange, and yellow, respectively. 


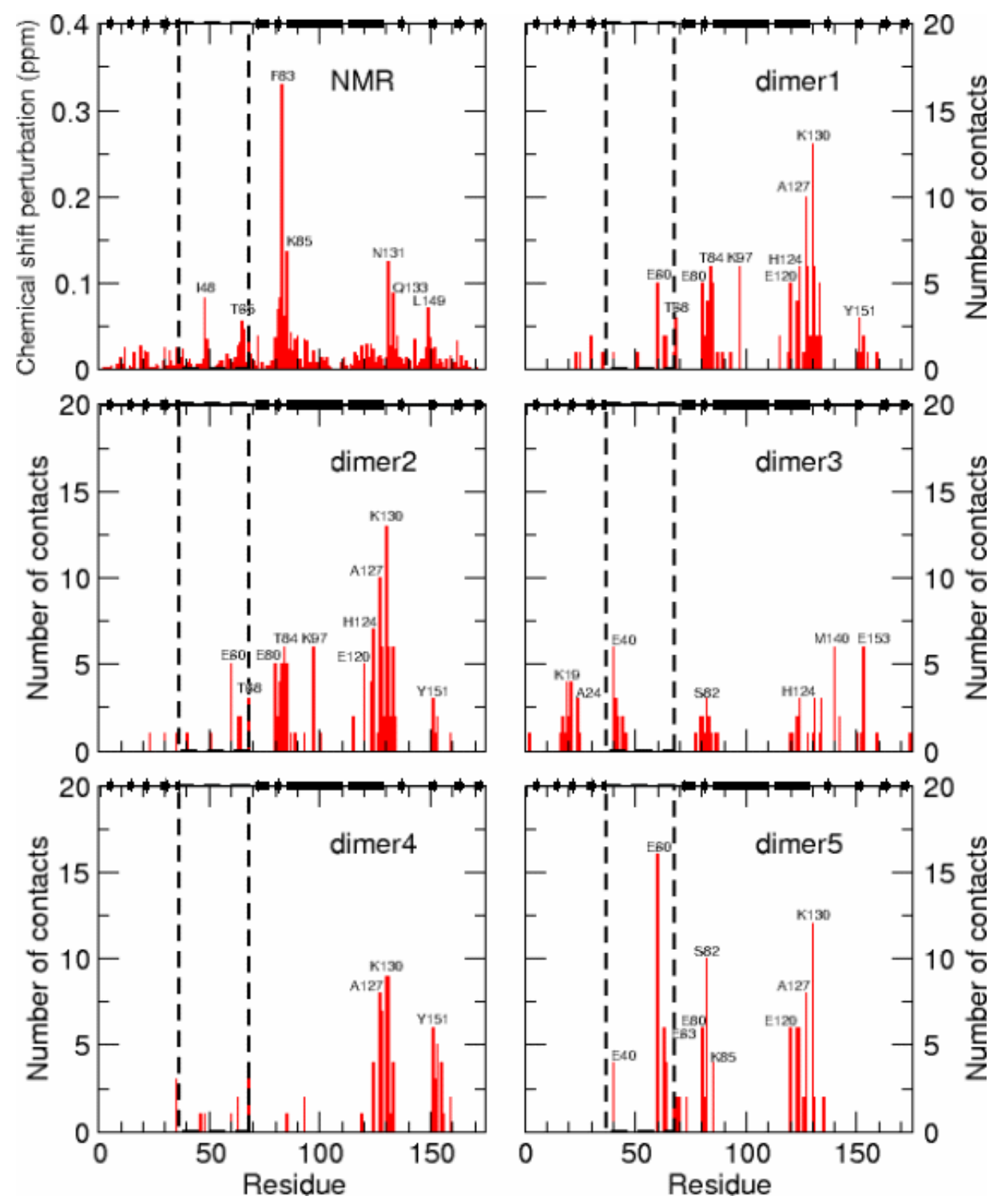

Figure 9: Top left: ${ }^{1} \mathrm{H}^{-15} \mathrm{~N}$ chemical shift perturbations observed upon TCTP dilution from $1 \mathrm{mM}$ to $50 \mu \mathrm{M}$. Top right, middle, and bottom: Number of contacts made by TCTP residues in the five lowest energy complexes. 


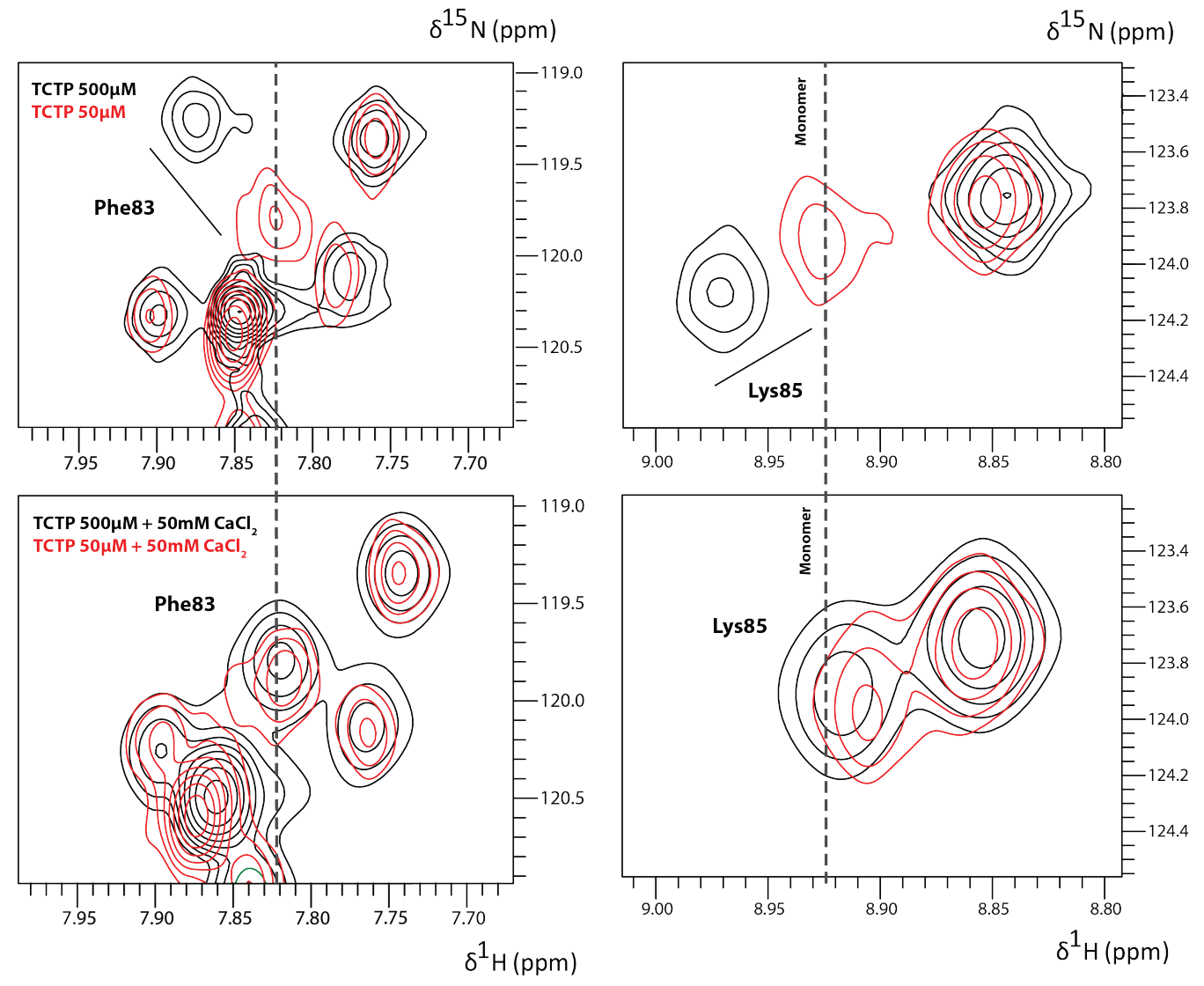

Figure 10: NMR cross-peaks of dimer-sensitive residues Phe83 (left) and Lys85 (right) at low $(50 \mu \mathrm{M})$ or medium $(500 \mu \mathrm{M})$ concentration of protein, without (top) or with $50 \mathrm{mM}$ calcium (bottom). The NMR spectra were otherwise collected in the buffer HEPES $50 \mathrm{mM}$ pH 7.4 $150 \mathrm{mM} \mathrm{NaCl} 2 \mathrm{mM}$ TCEP at $298 \mathrm{~K}$. 

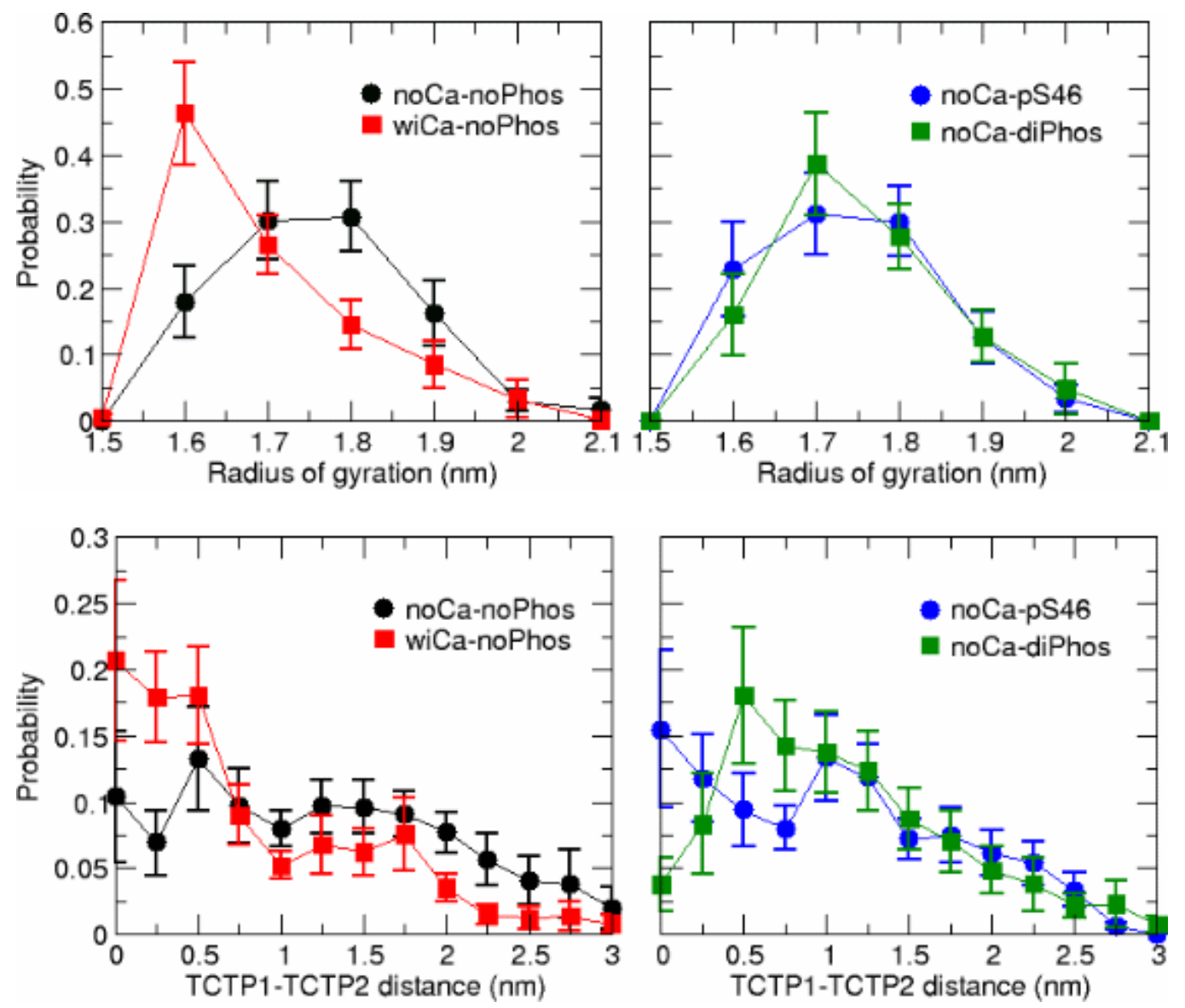

Figure 11: Probability distribtions of the TCTP radius of gyration and of the distance between the centers of mass of the two protein signatures TCTP1 and TCPT2. The mean values and standard deviations were calculated by using the bootstrap approach. 


\section{References}

[1] U.-A. Bommer, Cellular function and regulation of the translationally controlled tumour protein TCTP, Open Allergy J 5 (2012) 19-32.

[2] M. Nagano-Ito, S. Ichikawa, Biological Effects of Mammalian Translationally Controlled Tumor Protein (TCTP) on Cell Death, Proliferation, and Tumorigenesis, Biochemistry Research International 2012 (2012) 17.

[3] A. Telerman, R. Amson, The molecular programme of tumour reversion: the steps beyond malignant transformation, Nature Reviews Cancer 9 (2009) 206-216.

[4] R. Amson, S. Pece, J.-C. Marine, P. P. D. Fiore, A. Telerman, TPT1/ TCTP-regulated pathways in phenotypic reprogramming, Trends in Cell Biology 23 (2013) 37-46.

[5] J. Acunzo, V. Baylot, A. So, P. Rocchi, TCTP as therapeutic target in cancers, Cancer Treatment Reviews 40 (2014) 760-769.

[6] T. Kawakami, T. Ando, Y. Kawakami, HRF-interacting molecules, The Open Allergy Journal 5 (2012) 41-46.

[7] N. Assrir, F. Malard, E. Lescop, Structural Insights into TCTP and Its Interactions with Ligands and Proteins, in: A. Telerman, R. Amson (Eds.), TCTP/tpt1 - Remodeling Signaling from Stem Cell to Disease, Vol. 64, Springer International Publishing, 2017, pp. 9-46. 
[8] S. Li, M. Chen, Q. Xiong, J. Zhang, Z. Cui, F. Ge, Characterization of the Translationally Controlled Tumor Protein (TCTP) Interactome Reveals Novel Binding Partners in Human Cancer Cells, J. Proteome Res. 15 (2016) 3741-3751.

[9] J.-C. Sanchez, D. Schaller, F. Ravier, O. Golaz, S. Jaccoud, M. Belet, M. R. Wilkins, R. James, J. Deshusses, D. Hochstrasser, Translationally controlled tumor protein: a protein identified in several nontumoral cells including erythrocytes, Electrophoresis 18 (1997) 150-155.

[10] M. Kim, Y. Jung, K. Lee, C. Kim, Identification of the calcium binding sites in translationally controlled tumor protein, Arch. Pharm. Res. 23 (2000) 633-636.

[11] Y. Feng, D. Liu, H. Yao, J. Wang, Solution structure and mapping of a very weak calcium-binding site of human translationally controlled tumor protein by NMR, Archives of Biochemistry and Biophysics 467 (2007) 48-57.

[12] P. Graidist, M. Yazawa, M. Tonganunt, A. Nakatomi, C. C.-J. Lin, J.-Y. Chang, A. Phongdara, K. Fujise, Fortilin binds Ca2+ and blocks Ca2+dependent apoptosis in vivo, Biochemical Journal 408 (2007) 181-191.

[13] P. Thaw, N. J. Baxter, A. M. Hounslow, C. Price, J. P. Waltho, C. J. Craven, Structure of TCTP reveals unexpected relationship with guanine nucleotide-free chaperones, Nat Struct Mol Biol 8 (2001) 701-704.

[14] O. F. Lange, P. Rossi, N. G. Sgourakis, Y. Song, H.-W. Lee, J. M. Aramini, A. Ertekin, R. Xiao, T. B. Acton, G. T. Montelione, D. Baker, 
Determination of solution structures of proteins up to $40 \mathrm{kDa}$ using CSRosetta with sparse NMR data from deuterated samples, Proceedings of the National Academy of Sciences 109 (2012) 10873-10878.

[15] N. Dephoure, C. Zhou, J. Villén, S. A. Beausoleil, C. E. Bakalarski, S. J. Elledge, S. P. Gygi, A quantitative atlas of mitotic phosphorylation, Proceedings of the National Academy of Sciences 105 (2008) 1076210767.

[16] M. Fuxreiter, I. Simon, P. Friedrich, P. Tompa, Preformed Structural Elements Feature in Partner Recognition by Intrinsically Unstructured Proteins, Journal of Molecular Biology 338 (2004) 1015-1026.

[17] A. Mohan, C. J. Oldfield, P. Radivojac, V. Vacic, M. S. Cortese, A. K. Dunker, V. N. Uversky, Analysis of Molecular Recognition Features (MoRFs), Journal of Molecular Biology 362 (2006) 1043-1059.

[18] D. D. Boehr, R. Nussinov, P. E. Wright, The role of dynamic conformational ensembles in biomolecular recognition, Nature Chemical Biology 5 (2009) 789-796.

[19] H. R. A. Jonker, R. W. Wechselberger, R. Boelens, R. Kaptein, G. E. Folkers, The Intrinsically Unstructured Domain of PC4 Modulates the Activity of the Structured Core through Inter- and Intramolecular Interactions, Biochemistry 45 (2006) 5067-5081.

[20] K. Stott, M. Watson, F. S. Howe, J. G. Grossmann, J. O. Thomas, TailMediated Collapse of HMGB1 Is Dynamic and Occurs via Differential 
Binding of the Acidic Tail to the A and B Domains, Journal of Molecular Biology 403 (2010) 706-722.

[21] H.-Y. Li, J. Wang, F. Meng, Z.-K. Jia, Y. Su, Q.-F. Bai, L.-L. Lv, F.R. Ma, L. A. Potempa, Y.-B. Yan, S.-R. Ji, Y. Wu, An Intrinsically Disordered Motif Mediates Diverse Actions of Monomeric C-reactive Protein, Journal of Biological Chemistry 291 (2016) 8795-8804.

[22] F. Yarm, Plk Phosphorylation Regulates the Microtubule-Stabilizing Protein TCTP, Molecular and Cellular Biology 22 (2002) 6209-6221.

[23] Z. Solyom, M. Schwarten, L. Geist, R. Konrat, D. Willbold, B. Brutscher, Best-trosy experiments for time-efficient sequential resonance assignment of large disordered proteins, Journal of Biomolecular NMR 55 (2013) 311-321.

[24] L. Susini, S. Besse, D. Duflaut, A. Lespagnol, C. Beekman, G. Fiucci, A. Atkinson, D. Busso, P. Poussin, J. Marine, J. Martinou, J. Cavarelli, D. Moras, R. Amson, A. Telerman, TCTP protects from apoptotic cell death by antagonizing bax function, Cell Death and Differentiation 15 (2008) 1211-1220.

[25] G. Lipari, A. Szabo, Model-Free Approach to the Interpretation of Nuclear Magnetic Resonance Relaxation in Macromolecules. 1. Theory and Range of Validity, Journal of the American Chemical Society 104 (1982) $4546-4559$.

[26] J. Garcia de la Torre, M. Huertas, B. Carrasco, HYDRONMR: Prediction of NMR Relaxation of Globular Proteins from Atomic-Level Struc- 
tures and Hydrodynamic Calculations, Journal of Magnetic Resonance 147 (2000) 138-146.

[27] J. A. Marsh, V. K. Singh, Z. Jia, J. D. Forman-Kay, Sensitivity of secondary structure propensities to sequence differences between $\alpha$ - and $\gamma$ synuclein: Implications for fibrillation, Protein Science 15 (2006) 27952804.

[28] Y. Gachet, S. Tournier, M. Lee, A. Lazaris-Karatzas, T. Poulton, U. A. Bommer, The growth-related, translationally controlled protein P23 has properties of a tubulin binding protein and associates transiently with microtubules during the cell cycle, Journal of Cell Science 112 (1999) $1257-1271$.

[29] F. Bazile, A. Pascal, I. Arnal, C. Le Clainche, F. Chesnel, J. Kubiak, Complex relationship between TCTP, microtubules and actin microfilaments regulates cell shape in normal and cancer cells, Carcinogenesis 30 (2009) 555-565.

[30] M. Lucibello, S. Adanti, E. Antelmi, D. Dezi, S. Ciafre, M. Carcangiu, M. Zonfrillo, G. Nicotera, L. Sica, F. De Braud, P. Pierimarchi, PhosphoTCTP as a therapeutic target of dihydroartemisinin for aggressive breast cancer cells, Oncotarget 6 (2015) 5275-5291.

[31] H. Jeon, S. You, Y. Park, J. Chang, J. Kim, J. Oh, TCTP regulates spindle microtubule dynamics by stabilizing polar microtubules during mouse oocyte meiosis, Biochimica et Biophysica Acta 1863 (2016) 630637. 
[32] U. Cucchi, L. M. Gianellini, A. De Ponti, F. Sola, R. Alzani, V. Patton, A. Pezzoni, S. Troiani, M. B. Saccardo, S. Rizzi, others, Phosphorylation of TCTP as a marker for polo-like kinase-1 activity in vivo, Anticancer Research 30 (2010) 4973-4985.

[33] R. Amson, S. Pece, A. Lespagnol, R. Vyas, G. Mazzarol, D. Tosoni, I. Colaluca, G. Viale, S. Rodrigues-Ferreira, J. Wynendaele, O. Chaloin, J. Hoebeke, J.-C. Marine, P. P. Di Fiore, A. Telerman, Reciprocal repression between P53 and TCTP, Nature Medicine 18 (2011) 91-99.

[34] G. Funston, W. Goh, S. J. Wei, Q. S. Tng, C. Brown, L. Jiah Tong, C. Verma, D. Lane, F. Ghadessy, Binding of Translationally Controlled Tumour Protein to the N-Terminal Domain of HDM2 Is Inhibited by Nutlin-3, PLoS ONE 7 (2012) e42642.

[35] T. Yoon, J. Jung, M. Kim, K. M. Lee, E. C. Choi, K. Lee, Identification of the Self-Interaction of Rat TCTP/IgE-Dependent HistamineReleasing Factor Using Yeast Two-Hybrid System, Archives of Biochemistry and Biophysics 384 (2000) 379-382.

[36] J. Bhisutthibhan, S. Meshnick, Immunoprecipitation of $[3 \mathrm{H}]$ dihydroartemisinin translationally controlled tumor protein (TCTP) adducts from plasmodium falciparum-infected erythrocytes by using anti-TCTP antibodies, Antimicrobial Agents and Chemotherapy 45 (2001) 2397-2399.

[37] M. Gnanasekar, K. Rao, L. Chen, R. Narayanan, M. Geetha, A. Scott, K. Ramaswamy, P. Kaliraj, Molecular characterization of a calcium 
binding translationally controlled tumor protein homologue from the filarial parasites brugia malayi and wuchereria bancrofti, Molecular and Biochemical Parasitology 121 (2002) 107-118.

[38] M. Kim, H. J. Min, H. Y. Won, H. Park, J.-C. Lee, H.-W. Park, J. Chung, E. S. Hwang, K. Lee, Dimerization of translationally controlled tumor protein is essential for its cytokine-like activity, PLoS ONE 4 (2009) e6464.

[39] A. Lucas, X. Fu, J. Liu, M. Brannon, J. Yang, D. Capelluto, C. Finkielstein, Ligand binding reveals a role for heme in translationally-controlled tumor protein dimerization, PLoS ONE 9 (2014) e112823.

[40] S. Thébault, M. Agez, X. Chi, J. Stojko, V. Cura, S. B. Telerman, L. Maillet, F. Gautier, I. Billas-Massobrio, C. Birck, N. Troffer-Charlier, T. Karafin, J. Honoré, A. Senff-Ribeiro, S. Montessuit, C. M. Johnson, P. Juin, S. Cianférani, J.-C. Martinou, D. W. Andrews, R. Amson, A. Telerman, J. Cavarelli, TCTP contains a BH3-like domain, which instead of inhibiting, activates Bcl-xL, Scientific Reports 6 (2016) 19725.

[41] X. Dong, B. Yang, Y. Li, C. Zhong, J. Ding, Molecular basis of the acceleration of the GDP-GTP exchange of human Ras homolog enriched in brain by human translationally controlled tumor protein, Journal of Biological Chemistry 284 (2009) 23754-23764.

[42] K. A. Doré, J. ichi Kashiwakura, J. M. McDonnell, H. J. Gould, T. Kawakami, B. J. Sutton, A. M. Davies, Crystal structures of murine and human histamine-releasing factor (hrf/tctp) and a model for hrf 
dimerisation in mast cell activation, Molecular Immunology 93 (2018) $216-222$.

[43] M. Sattler, J. Schleucher, C. Griesinger, Heteronuclear multidimensional NMR experiments for the structure determination of proteins in solution employing pulsed field gradients, Progress in Nuclear Magnetic Resonance Spectroscopy 34 (1999) 93-158.

[44] W. Vranken, W. Boucher, T. Stevens, R. Fogh, A. Pajon, M. Llinas, E. Ulrich, J. Markley, J. Ionides, E. Laue, The CCPN data model for NMR spectroscopy: development of a software pipeline, Proteins 59 (2005) 687-696.

[45] G. W. Vuister, A. Bax, Quantitative J correlation: a new approach for measuring homonuclear three-bond $\mathrm{J}(\mathrm{HNH} \alpha)$ coupling constants in 15N-enriched proteins, Journal of the American Chemical Society 115 (1993) $7772-7777$.

[46] P. Schanda, B. Brutscher, Very fast two-dimensional NMR spectroscopy for real-time investigation of dynamic events in proetins on the time scale of seconds, Journal of the American Chemical Society 127 (2005) 8014-8015.

[47] F. Delaglio, S. Grzesiek, G. W. Vuister, G. Zhu, J. Pfeifer, A. Bax, NMRPipe: A multidimensional spectral processing system based on UNIX pipes, Journal of Biomolecular NMR 6 (3) (1995) 277-293.

[48] P. Dosset, J.-C. Hus, M. Blackledge, D. Marion, Efficient analysis of 
macromolecular rotational diffusion from heternuclear relaxation data, J. Biomol. NMR 16 (2000) 23-28.

[49] E. F. Pettersen, T. D. Goddard, C. C. Huang, G. S. Couch, D. M. Greenblatt, E. C. Meng, T. E. Ferrin, UCSF Chimera?A visualization system for exploratory research and analysis, Journal of Computational Chemistry 25 (2004) 1605-1612.

[50] D. Van Der Spoel, E. Lindahl, B. Hess, G. Groenhof, A. E. Mark, H. J. C. Berendsen, GROMACS: Fast, flexible, and free, J. Comput. Chem. 26 (2005) 1701-1718.

[51] B. Hess, C. Kutzner, D. van der Spoel, E. Lindahl, GROMACS 4: Algorithms for Highly Efficient, Load-Balanced, and Scalable Molecular Simulation, Journal of Chemical Theory and Computation 4 (2008) 435447.

[52] K. Lindorff-Larsen, S. Piana, K. Palmo, P. Maragakis, J. L. Klepeis, R. O. Dror, D. E. Shaw, Improved side-chain torsion potentials for the Amber ff99sb protein force field, Proteins 78 (2010) 1950-1958.

[53] W. L. Jorgensen, J. Chandrasekhar, J. D. Madura, R. W. Impey, M. L. Klein, Comparison of simple potential functions for simulating liquid water, Journal of Chemical Physics 79 (1983) 926-935.

[54] U. Essmann, L. Perera, M. L. Berkowitz, T. Darden, H. Lee, L. G. Pedersen, A smooth particle mesh Ewald method, Journal of chemical physics 103 (1995) 8577-8593. 
[55] B. Hess, P-LINCS: A Parallel Linear Constraint Solver for Molecular Simulation, Journal of Chemical Theory and Computation 4 (2008) 116122.

[56] S. Miyamoto, P. A. Kollman, SETTLE: an analytical version of the SHAKE and RATTLE algorithm for rigid water models, Journal of computational chemistry 13 (1992) 952-962.

[57] S. Nosé, A unified formulation of the constant temperature molecular dynamics methods, Journal of Chemical Physics 81 (1984) 511-519.

[58] W. G. Hoover, Canonical dynamics: Equilibrium phase-space distributions, Physical Review A 31 (1985) 1695-1697.

[59] M. Parrinello, A. Rahman, Polymorphic transitions in single crystals: A new molecular dynamics method, Journal of Applied Physics 52 (1981) $7182-7190$.

[60] K. Ösapay, D. A. Case, Analysis of proton chemical shifts in regular secondary structure of proteins, Journal of biomolecular NMR 4 (1994) $215-230$.

[61] X.-P. Xu, D. A. Case, Automated prediction of $15 \mathrm{~N}, 13 \mathrm{C} \alpha, 13 \mathrm{C} \beta$ and 13C' chemical shifts in proteins using a density functional database, Journal of biomolecular NMR 21 (2001) 321-333.

[62] M. Karplus, Contact electron-spin coupling of nuclear magnetic moments, Journal of Chemical Physics 30 (1959) 11-15. 
[63] A. Saladin, S. Fiorucci, P. Poulain, C. Prévost, M. Zacharias, PTools: an opensource molecular docking library, BMC Structural Biology 9 (2009) 27.

[64] N. Basdevant, D. Borgis, T. Ha-Duong, A coarse-grained proteinprotein potential derived from an all-atom force field, Journal of Physical Chemistry B 111 (2007) 9390-9399.

[65] N. Basdevant, D. Borgis, T. Ha-Duong, Modeling protein-protein recognition in solution using the coarse-grained force field SCORPION, Journal of Chemical Theory and Computation 9 (2013) 803-813. 